DRAFT VERSION APRIL 9, 2018

Preprint typeset using LATEX style AASTeX6 v. 1.0

\title{
ON THE RATE AND ON THE GRAVITATIONAL WAVE EMISSION OF SHORT AND LONG GRBS
}

\author{
R. Ruffini ${ }^{1,2,3,4}$, J. Rodriguez ${ }^{1,2}$, M. Muccino ${ }^{1,2}$, J. A. Rueda ${ }^{1,2,4}$, Y. Aimuratov ${ }^{1,2}$, U. Barres De Almeida ${ }^{4,5}$, \\ L. Becerra ${ }^{1,2}$, C. L. Bianco ${ }^{1,2}$, C. Cherubini ${ }^{6,7}$, S. Filippi ${ }^{6,7}$, D. Gizzi ${ }^{1}$, M. Kovacevic ${ }^{1,2,3}$, R. Moradi ${ }^{1,2}$, \\ F. G. Oliveira ${ }^{1,2,3}$, G. B. Pisani ${ }^{1,2}$, Y. WAnG ${ }^{1,2}$
}

(Dated: April 9, 2018)

\footnotetext{
${ }^{1}$ Dipartimento di Fisica and ICRA, Sapienza Università di Roma, P.le Aldo Moro 5, I-00185 Rome, Italy

${ }^{2}$ ICRANet, P.zza della Repubblica 10, I-65122 Pescara, Italy

${ }^{3}$ Université de Nice Sophia Antipolis, CEDEX 2, Grand Château Parc Valrose, Nice, France

${ }^{4}$ ICRANet-Rio, Centro Brasileiro de Pesquisas Físicas, Rua Dr. Xavier Sigaud 150, 22290-180 Rio de Janeiro, Brazil

${ }^{5}$ Centro Brasileiro de Pesquisas Físicas, Rua Dr. Xavier Sigaud 150, 22290-180 Rio de Janeiro, Brazil

${ }^{6}$ Unit of Nonlinear Physics and Mathematical Modeling, Università Campus Bio-Medico di Roma, Via A. del Portillo 21, I-00128 Rome, Italy

${ }^{7}$ ICRA, Università Campus Bio-Medico di Roma, Via A. del Portillo 21, I-00128 Rome, Italy
}

\section{ABSTRACT}

On the ground of the large number of gamma-ray bursts (GRBs) detected with cosmological redshift, we have introduced a new classification of GRBs in seven subclasses, all with binary progenitors originating gravitational waves (GWs). Each binary is composed by a different combination of carbonoxygen cores $\left(\mathrm{CO}_{\text {core }}\right)$, neutron stars (NSs), black holes (BHs) and white dwarfs (WDs). This opens an ample new scenario for the role of GWs both as detectable sources and as a determining factor in the coalescence process of the GRB binary progenitors. The long bursts, traditionally assumed to originate from a single $\mathrm{BH}$ with an ultra-relativistic jetted emission, not expected to emit GWs, have instead been subclassified as (I) X-ray flashes (XRFs), (II) binary-driven hypernovae (BdHNe), and (III) BH-supernovae (BH-SNe). They are framed within the induced gravitational collapse (IGC) paradigm with progenitor a tight binary composed of a $\mathrm{CO}_{\text {core }}$ and a $\mathrm{NS}$ or $\mathrm{BH}$ companion. The supernova $(\mathrm{SN})$ explosion of the $\mathrm{CO}_{\text {core }}$ triggers a hypercritical accretion process onto the companion NS or BH. If the accretion is not sufficient for the NS to reach its critical mass, an XRF occurs, while when the $\mathrm{BH}$ is already present or formed by the hypercritical accretion, a BdHN occurs. In the case these binaries are not disrupted by the mass-loss process, XRFs lead to NS-NS binaries and BdHNe lead to NS-BH ones. The short bursts, originating in NS-NS mergers, are subclassified as (IV) short gamma-ray flashes (S-GRFs) and (V) short GRBs (S-GRBs), the latter when a BH is formed. Two additional families are (VI) ultra-short GRBs (U-GRBs) and (VII) gamma-ray flashes (GRFs), respectively formed in NS-BH and NS-WD mergers. We use the estimated occurrence rate of the above subclasses and their GW emission to assess their detectability by Advanced LIGO and Virgo, eLISA, and resonant bars. We also discuss the consequences of our results in view of the recent announcement of the LIGO-Virgo Collaboration of the source GW 170817 as being originated by a NS-NS merger.

Keywords: gamma-ray burst: general — gravitational waves — black hole physics — stars: neutron — white dwarfs — binaries: general

\section{INTRODUCTION}

Thanks to the extensive observations carried out by $\gamma$-ray telescopes, such as AGILE, BATSE, BeppoSAX, Fermi, HETE-II, INTEGRAL, Konus/WIND and Swift, our understanding of "long" and "short" gamma-ray burst (GRB) progenitor systems has greatly improved. This has led also to a vast literature devoted to the estimate of their relative occurrence rates, all in gen- eral agreement. For long bursts see, e.g., Soderberg et al. (2006a); Guetta \& Della Valle (2007); Liang et al. (2007); Virgili et al. (2009); Rangel Lemos et al. (2010); Wanderman \& Piran (2010); Guetta et al. (2011); Kovacevic et al. (2014); for short bursts see, e.g., Virgili et al. (2011); Wanderman \& Piran (2015); and for both long and short bursts see, e.g., Sun et al. (2015); Ruffini et al. (2016b). The rates of GW emission from GRBs have been calculated in the literature at a time 
in which short GRBs were considered to be originated in neutron star-neutron star (NS-NS) binaries, while long GRBs, were considered to be originated in single events $^{1}$, e.g. collapsars (Woosley 1993; MacFadyen \& Woosley 1999; MacFadyen et al. 2001; Woosley \& Bloom 2006; see however Ruffini et al. 2018a) and magnetars (Usov 1992; Dai \& Lu 1998a,b; Kluźniak \& Ruderman 1998; Zhang \& Mészáros 2001; see however Ruffini et al. 2016b). Thus, only short GRBs have been up to now considered to estimate the simultaneous detection rate of gravitational waves (GWs) and GRBs. For instance, Wanderman \& Piran (2015) used the luminosity function of short GRBs observed by Swift; Yonetoku et al. (2014) by BATSE; Patricelli et al. (2016) by Fermi and Ghirlanda et al. (2016) by Swift and Fermi.

In our recent works (see Ruffini et al. 2016b, and references therein) we have introduced a new classification in which all GRBs, namely both long and short, originate from merging and/or accreting binary systems, each composed by a different combination of carbonoxygen cores $\left(\mathrm{CO}_{\text {core }}\right)$, NSs, black holes (BHs) and white dwarfs (WDs). For each system the initial state and the final state are respectively here referred to as "in-state" and "out-state". This opens an ample new scenario for the role of GWs both as detectable sources and as a determining factor in the coalescence process of the GRB progenitors.

We interpret the traditional long GRBs within the induced gravitational collapse (IGC) paradigm (Ruffini et al. 2006, 2007, 2008; Izzo et al. 2012a; Rueda \& Ruffini 2012; Fryer et al. 2014; Ruffini et al. 2015a) that proposes as in-state a tight binary system composed of a $\mathrm{CO}_{\text {core }}$ undergoing a supernova ( $\mathrm{SN}$ ) explosion and a companion compact object, e.g. a NS (or a BH). The SN explosion triggers a hypercritical accretion onto the NS companion, whose details has been studied, simulated and presented in several publications (see, e.g., Becerra et al. 2016; Fryer et al. 2015b; Becerra et al. 2015; Fryer et al. 2014, references therein and Appendix A). Depending upon the binary parameters, the hypercritical accretion can lead to three very different outcomes:

I. X-ray flashes (XRFs) with isotropic energy $E_{\text {iso }} \lesssim$ $10^{52}$ erg and rest-frame spectral peak energy $E_{p, i} \lesssim 200 \mathrm{keV}$. This class occurs in $\mathrm{CO}_{\text {core }}-\mathrm{NS}$ binaries when the hypercritical accretion onto the NS companion is not enough to induce gravitational collapse into a BH (Becerra et al. 2016, 2015). Following this definition, Ruffini et al. (2016b) estimated for the XRF a local observed

\footnotetext{
${ }^{1}$ With the exception of the binary progenitors proposed in Fryer \& Woosley (1998); Fryer et al. (1999a,b); Belczynski et al. (2002).
}

rate of $\rho_{\mathrm{XRF}}=100_{-34}^{+45} \mathrm{Gpc}^{-3} \mathrm{yr}^{-1}$ (Ruffini et al. $2016 \mathrm{~b})$. This rate is in agreement with that of low-luminous long GRBs, e.g.:325 $-177 \mathrm{Gpc}^{-3} \mathrm{yr}^{-1}$ (Liang et al. 2007), $200 \mathrm{Gpc}^{-3} \mathrm{yr}^{-1}$ (Virgili et al. 2009), $164_{-65}^{+98} \mathrm{Gpc}^{-3} \mathrm{yr}^{-1}$ (Sun et al. 2015). After the SN explosion the binary can either get disrupted or remain bound depending upon the mass loss and/or natal kick imparted to the system (see Postnov \& Yungelson 2014, references therein and Appendix A.5). In the former case the XRF leads to two runaway NSs, while in the latter one, the out-states of XRFs are binaries composed of a newly-formed $\sim 1.4-1.5 M_{\odot}$ NS (hereafter $\nu \mathrm{NS}$ ) born in the SN explosion, and a massive NS (MNS) which accreted matter from the SN ejecta. Typical periods of these binaries are $P_{\text {orb }} \gtrsim 30 \mathrm{~min}$ (Becerra et al. 2016).

II. Binary-driven hypernovae (BdHNe) with $E_{\text {iso }} \gtrsim$ $10^{52} \mathrm{erg}$ and $E_{p, i} \gtrsim 200 \mathrm{keV}$. BdHNe occur in more compact $\mathrm{CO}_{\text {core }}-\mathrm{NS}$ binaries which leads to a more massive hypercritical accretion onto the NS, hence leading to $\mathrm{BH}$ formation. Following this definition, Ruffini et al. (2016b) estimated for the BdHNe a local observed rate $\rho_{\mathrm{BdHN}}=0.77_{-0.08}^{+0.09} \mathrm{Gpc}^{-3} \mathrm{yr}^{-1}$ (Ruffini et al. 2016b). This rate is in agreement with that for high-luminous long GRBs, e.g.: $1.3_{-0.7}^{+0.6} \mathrm{Gpc}^{-3} \mathrm{yr}^{-1}$ (Wanderman \& Piran 2010) and $0.8_{-0.1}^{+0.1} \mathrm{Gpc}^{-3} \mathrm{yr}^{-1}$ (Sun et al. 2015). As in the case of XRFs, the SN explosion can disrupt the binary depending upon the mass loss and/or natal kick. In the case when the system remains bound, the out-states of BdHNe are $\nu \mathrm{NS}-\mathrm{BH}$ binaries (see Fryer et al. 2015b, and Appendix A.5). Typical periods of these binaries are $5 \mathrm{~min} \lesssim P_{\text {orb }} \lesssim 30 \mathrm{~min}$ (Becerra et al. 2016).

III. BH-SN with $E_{\text {iso }} \gtrsim 10^{54}$ erg and $E_{p, i} \gtrsim 2 \mathrm{MeV}$. $\mathrm{BH}-\mathrm{SN}$ occur in close $\mathrm{CO}_{\text {core }}$ (or Helium or WolfRayet star)-BH binaries (Ruffini et al. 2001) in which the hypercritical accretion occurs onto a previously formed BH. Such BH-SN systems correspond to the late evolutionary stages of X-ray binaries as Cyg X-1 (Giacconi \& Ruffini 1978; Belczynski et al. 2011), or microquasars (Mirabel \& Rodríguez 1998). These systems might be also formed following the binary evolutionary patch leading to the scenario XI in Fryer et al. (1999a). Since the estimated rate of $\mathrm{BdHNe}$ covers systems with the above $E_{\text {iso }}$ and $E_{p, i}$ range, we can adopt the rate of $\mathrm{BdHNe}$ as an upper limit to the rate of $\mathrm{BH}-\mathrm{SNe}$, i.e. $\rho_{\mathrm{BH}-\mathrm{SN}} \lesssim \rho_{\mathrm{BdHN}}=$ $0.77_{-0.08}^{+0.09} \mathrm{Gpc}^{-3} \mathrm{yr}^{-1}$ (Ruffini et al. 2016b). As in the above cases of XRFs and BdHNe, the SN explosion may disrupt the binary. If the binary 
Table 1. Summary of the astrophysical aspects of the different GRB subclasses and of their observational properties. In the first four columns we indicate the GRB subclasses and their corresponding in-states and the out-states. In columns $5-8$ we list the ranges of $E_{\mathrm{p}, \mathrm{i}}$ and $E_{\text {iso }}$ (rest-frame $\left.1-10^{4} \mathrm{keV}\right), E_{\mathrm{iso}, \mathrm{X}}$ (rest-frame $0.3-10 \mathrm{keV}$ ), and $E_{\text {iso, GeV }}$ (rest-frame $0.1-100 \mathrm{GeV}$ ). Columns 9 and 10 list, for each GRB subclass, the maximum observed redshift and the local observed rate $\rho_{\text {GRB }}$ obtained in Ruffini et al. (2016b). We refer the reader to Appendix B for details on the method used to calculate $\rho_{\text {GRB }}$.

\begin{tabular}{|c|c|c|c|c|c|c|c|c|c|}
\hline & subclass & In-state & Out-state & $\begin{array}{c}E_{\mathrm{p}, \mathrm{i}} \\
(\mathrm{MeV})\end{array}$ & $\begin{array}{l}E_{\text {iso }} \\
(\mathrm{erg})\end{array}$ & $\begin{array}{c}E_{\text {iso,X }} \\
(\mathrm{erg})\end{array}$ & $\begin{array}{c}E_{\text {iso,Gev }} \\
(\mathrm{erg})\end{array}$ & $z_{\max }$ & $\begin{array}{c}\rho_{\mathrm{GRB}} \\
\left(\mathrm{Gpc}^{-3} \mathrm{yr}^{-1}\right)\end{array}$ \\
\hline $\mathrm{I}$ & XRFs & $\mathrm{CO}_{\text {core }}-\mathrm{NS}$ & $\nu \mathrm{NS}-\mathrm{NS}$ & $\lesssim 0.2$ & $\sim 10^{48}-10^{52}$ & $\sim 10^{48}-10^{51}$ & - & 1.096 & $100_{-34}^{+45}$ \\
\hline II & $\mathrm{BdHNe}$ & $\mathrm{CO}_{\text {core }}-\mathrm{NS}$ & $\nu \mathrm{NS}-\mathrm{BH}$ & $\sim 0.2-2$ & $\sim 10^{52}-10^{54}$ & $\sim 10^{51}-10^{52}$ & $\lesssim 10^{53}$ & 9.3 & $0.77_{-0.08}^{+0.09}$ \\
\hline III & BH-SN & $\mathrm{CO}_{\text {core }}-\mathrm{BH}$ & $\nu \mathrm{NS}-\mathrm{BH}$ & $\gtrsim 2$ & $>10^{54}$ & $\sim 10^{51}-10^{52}$ & $\gtrsim 10^{53}$ & 9.3 & $\lesssim 0.77_{-0.08}^{+0.09}$ \\
\hline IV & S-GRFs & NS-NS & MNS & $\lesssim 2$ & $\sim 10^{49}-10^{52}$ & $\sim 10^{49}-10^{51}$ & - & 2.609 & $3.6_{-1.0}^{+1.4}$ \\
\hline V & S-GRBs & NS-NS & $\mathrm{BH}$ & $\gtrsim 2$ & $\sim 10^{52}-10^{53}$ & $\lesssim 10^{51}$ & $\sim 10^{52}-10^{53}$ & 5.52 & $\left(1.9_{-1.1}^{+1.8}\right) \times 10^{-3}$ \\
\hline VI & U-GRBs & $\nu \mathrm{NS}-\mathrm{BH}$ & $\mathrm{BH}$ & $\gtrsim 2$ & $>10^{52}$ & - & - & - & $\gtrsim 0.77_{-0.08}^{+0.09}$ \\
\hline VII & GRFs & NS-WD & MNS & $\sim 0.2-2$ & $\sim 10^{51}-10^{52}$ & $\sim 10^{49}-10^{50}$ & - & 2.31 & $1.02_{-0.46}^{+0.71}$ \\
\hline
\end{tabular}

survives, then the out-states of BH-SNe can be a $\nu$ NS-BH or a BH-BH if the SN central remnant directly collapses to a BH. However, the latter scenario is currently ruled out by the observations of pre-SN cores which appear to have masses $\lesssim 18 M_{\odot}$, very low to lead to direct $\mathrm{BH}$ formation (see, e.g., Smartt 2009, 2015, for details).

In the current literature such a difference between an $\mathrm{XRF}$, a BdHN and a BH-SN in the evaluation of GWs, here implemented, is still missing.

We turn now to the short bursts. Although their progenitors are still under debate, there is an ample consensus in the scientific community that they originate from NS-NS and/or NS-BH merging binaries (see, e.g., Goodman 1986; Paczynski 1986; Eichler et al. 1989; Narayan et al. 1991; Meszaros \& Rees 1997; Rosswog et al. 2003; Lee et al. 2004; Berger 2014). By adopting the same instates as in the above traditional models, namely NSNS and/or NS-BH mergers, they can be divided into three subclasses (Fryer et al. 2015b; Ruffini et al. 2015b, 2016b):

IV. Short gamma-ray flashes (S-GRFs), with $E_{\text {iso }} \lesssim$ $10^{52}$ erg and $E_{p, i} \lesssim 2 \mathrm{MeV}$, occur when no $\mathrm{BH}$ is formed in the NS-NS merger, i.e. they lead to a MNS. Following this definition, Ruffini et al. (2016b) estimated for the S-GRFs a local observed rate $\rho_{\mathrm{S}-\mathrm{GRF}}=3.6_{-1.0}^{+1.4} \mathrm{Gpc}^{-3} \mathrm{yr}^{-1}$.

V. Authentic short GRBs (S-GRBs), with $E_{\text {iso }} \gtrsim$ $10^{52} \mathrm{erg}$ and $E_{p, i} \gtrsim 2 \mathrm{MeV}$, occur when a $\mathrm{BH}$ is formed in the NS-NS merger (Ruffini et al. 2016a, 2015b; Muccino et al. 2013). Following this definition, Ruffini et al. (2016b) estimated for the S-GRBs a local observed rate $\rho_{\mathrm{S}-\mathrm{GRB}}=$ $\left(1.9_{-1.1}^{+1.8}\right) \times 10^{-3} \mathrm{Gpc}^{-3} \mathrm{yr}^{-1}$ (Ruffini et al. 2016b).

VI. Ultra-short GRBs (U-GRBs), a new subclass of short bursts originating from $\nu \mathrm{NS}-\mathrm{BH}$ merging binaries. They can originate from BdHNe (see II above) or from BH-SN events (see III above). Since in Fryer et al. (2015b) it was shown that the majority of BdHN out-states remain bound, we can assume as upper limit of their local density rate, $\rho_{\mathrm{U}-\mathrm{GRB}} \approx \rho_{\mathrm{BdHN}}=0.77_{-0.08}^{+0.09} \mathrm{Gpc}^{-3} \mathrm{yr}^{-1}$ (Ruffini et al. 2016b). U-GRBs are yet unobserved/unidentified and present a great challenge not only in the case of high-energy but also possibly in the radio band where they could manifest themselves, prior to the merger phase, as pulsarBH binaries (see, e.g., Tauris et al. 2015b, and references therein).

It is important to mention that the sum of the occurrence rates of the above short burst subclasses IV-VI is in agreement with the estimates obtained from the whole short burst population reported in the literature (see, e.g., Wanderman \& Piran 2015; Sun et al. 2015). It is then clear that what in the current literature are indicated as short GRBs are actually just S-GRFs.

In addition to the above three subclasses of long bursts and three subclasses of short bursts, we recall the existence of a class of bursts occurring in a low-density circumburst medium $(\mathrm{CBM})$, e.g. $n_{\mathrm{CBM}} \sim 10^{-3} \mathrm{~cm}^{-3}$, which show hybrid properties between short and long bursts in $\gamma$-rays. These bursts are not associated with $\mathrm{SNe}$, even at low redshift where the $\mathrm{SN}$ detection would not be precluded (Della Valle et al. 2006). We have called such bursts as Gamma-ray flashes (GRFs) (Ruffini et al. 2016b).

VII. GRFs have $10^{51} \lesssim E_{\text {iso }} \lesssim 10^{52}$ erg and $0.2 \lesssim$ $E_{p, i} \lesssim 2 \mathrm{MeV}$. These bursts, which shows an extended and softer emission, are thought to originate in NS-WD mergers (Ruffini et al. 2016b). NS-WD binaries are notoriously common astrophysical systems (Cadelano et al. 2015) and possible evolutionary scenarios leading to such mergers 
have been envisaged (see, e.g., Fryer et al. 1999b; Lazarus et al. 2014; Tauris et al. 2000) $)^{2}$ GRFs form a MNS and not a BH (see Ruffini et al. 2016b, for details). Following this definition, Ruffini et al. (2016b) estimated for the GRFs a local observed rate $\rho_{\mathrm{GRF}}=1.02_{-0.46}^{+0.71} \mathrm{Gpc}^{-3} \mathrm{yr}^{-1}$ (Ruffini et al. $2016 \mathrm{~b})$. This density rate appears to be low with respect to the current number of known NS-WD binaries in the Galaxy (see, e.g., Cadelano et al. 2015). From the GRB side, we note that indeed only one NS-WD merger has been identified (see analysis of GRB 060614 in Caito et al. 2009). The above implies that, very likely, the majority of the expected mergers are under the threshold of the existing $\mathrm{X}$ and gamma-ray detectors.

The aforementioned density rates for all GRB subclasses have been estimated in Ruffini et al. (2016b) assuming no beaming. The presence of beaming would require the observation of achromatic jet breaks in the afterglow light curve. In the present case of short bursts such clear achromatic jet breaks have never been observed. Fong et al. (2015) reported 4 measured jet breaks in a sample of 11 short bursts: GRB 051221A, GRB 090426A, GRB 111020A, GRB 130603B (see Table 5 there). However:

- GRB 051221A: The break is inferred only from the $\mathrm{X}$-ray light curve, while the contemporary optical and radio data does not support such an interpretation (see Soderberg et al. 2006b).

- GRB 090426A: The break is inferred from the optical band only, and there are no contemporary observations in other bands (see Nicuesa Guelbenzu et al. 2011).

- GRB 111020A: The break is inferred only from the $\mathrm{X}$-ray light curve, but this interpretation is based on a single upper limit by Chandra and no data points (see Fong et al. 2012).

- GRB 130603B: The break is inferred from the optical band and is compatible with the radio data. However, contemporary X-ray observations are clearly contradicting this interpretation and presents no break at all. In fact, the authors invoke the presence of an extra source to justify what they call "late time X-ray excess" (see Fong et al. 2014).

In addition, Aimuratov et al. (2017a); Ruffini et al. (2018b) have shown that, in all the identified S-GRBs,

2 An additional (but less likely) scenario leading to merging NS-WD systems might occur in a NS-NS approaching the merger phase (Ruffini et al. 2016b). According to Bildsten \& Cutler (1992) and Clark \& Eardley (1977) (see also references therein), in a very close, NS-NS binary with unequal-mass components, stable mass-transfer from the less massive to the more massive NS might occur for appropriate mass-ratios in such a way that the donor NS moves outward in the mass-loss process until it reaches the beta-decay instability becoming a low-mass WD.

\begin{tabular}{lc}
\hline \hline Extended wording & Acronym \\
\hline Binary-driven hypernova & BdHN \\
Black hole & BH \\
Carbon-oxygen core & $\mathrm{CO}_{\text {core }}$ \\
Gamma-ray burst & $\mathrm{GRB}$ \\
Gamma-ray flash & $\mathrm{GRF}$ \\
Induced gravitational collapse & $\mathrm{IGC}$ \\
Massive neutron star & $\mathrm{MNS}$ \\
Neutron star & $\mathrm{NS}$ \\
New neutron star created in the SN explosion & $\nu \mathrm{NS}$ \\
Short gamma-ray burst & $\mathrm{S}-\mathrm{GRB}$ \\
Short gamma-ray flash & $\mathrm{S}-\mathrm{GRF}$ \\
Supernova & $\mathrm{SN}$ \\
Ultrashort gamma-ray burst & $\mathrm{U}-\mathrm{GRB}$ \\
White dwarf & WD \\
X-ray flash & XRF \\
\hline
\end{tabular}

Table 2. Acronyms used in this work in alphabetic order.

the $\mathrm{GeV}$ emission has been always observed when the source was within the Fermi-LAT field of view. This result points as well to no significant presence of beaming in the GeV emission of S-GRBs.

Therefore, all the above points imply that there is still no evidence for the need to assume beaming.

We show in Table 1 a summary of the astrophysical aspects related to the GRB subclasses and their observational properties.

The aim of this article is to use the rate of occurrence of the above GRB subclasses to assess the detectability of their associated GW emission by the ground-based interferometers Advanced LIGO and Advanced Virgo, by the space-based interferometer eLISA, as well as by the resonant bars, for completeness.

We show in Table 2 a summary of acronyms used in this work.

\section{RELEVANCE OF THE NS STRUCTURE AND CRITICAL MASS}

Having introduced the above seven subclasses of GRBs, it becomes clear the relevance of the NS physics, in particular the NS critical mass value, in the definition of the subclasses I-II and IV-V.

First, we recall that in our previous works we have adopted a NS critical mass within the range 2.2-3.4 $M_{\odot}$, depending on the equation of state (EOS) and on the NS angular momentum (Cipolletta et al. 2015; Becerra et al. 2015; Belvedere et al. 2014). These quoted values are for EOS based on relativistic nuclear mean-field models (in this case the NL3, TM1 and GM1 models) and for a NS angular momentum from $J=0$ up to $J_{\max } \approx 0.7 G M^{2} / c$ (Cipolletta et al. 2015). Hereafter, we adopt the stiffest 
model, namely the NL3 EOS, which leads to the largest NS critical mass: from $M_{\text {crit }} \approx 2.7 M_{\odot}$ at $J=0$, that, as expected, is lower than the non-rotating critical mass upper limit of $3.2 M_{\odot}$ established by Rhoades \& Ruffini (1974), to $M_{\text {crit }} \approx 3.4 M_{\odot}$ at $J_{\max }$ (Cipolletta et al. 2015). Our choice of relativistic mean-field theory models is based on the fact that they satisfy important properties such as Lorentz covariance, relativistic self-consistency (hence they do not violate causality), intrinsic inclusion of spin, and a simple mechanism of nuclear matter saturation (see, e.g., Dutra et al. 2014, 2016, for further details on these kind of models). The above three representative EOS that we have explored satisfy in addition the astrophysical constraint of leading to a NS critical mass larger than the heaviest massive NS observed, PSR J0348+0432, with $M=2.01 \pm 0.04 M_{\odot}$ (Antoniadis et al. 2013).

As discussed in Ruffini et al. (2016b), the separatrix energy value of $\approx 10^{52} \mathrm{erg}$ between the subclasses I and II appears as a theoretical estimate of the upper limit to the energy emitted in the hypercritical accretion process onto $\mathrm{a} \sim 1.4 M_{\odot}$ NS (see, e.g., Becerra et al. 2016) and the afore-mentioned adopted critical mass. This has been shown to be in agreement with the observations of 20 XRFs and 233 BdHNe (up to the end of 2014). In fact, observationally, the current upper limit for XRFs is $(7.3 \pm 0.7) \times 10^{51} \mathrm{erg}$, and the lower limit for BdHNe is $(9.2 \pm 1.3) \times 10^{51} \mathrm{erg}$ (see Ruffini et al. 2016b, for further details). It is clear that the separatrix energy should have some dependence on the initial NS mass undergoing accretion and on the precise value of the non-rotating critical mass. Although the precise value of the latter is yet unknown, it is constrained within the range $2.0-3.2 M_{\odot}$, where the lower value is the mass of PSR J0348+0432, and the upper value is the wellestablished absolute maximum NS mass of Rhoades \& Ruffini (1974).

It is clear that similar arguments apply also to the case of the subclasses IV and V (Ruffini et al. 2015b); namely the amount of energy emitted during the NS-NS merger leading to a $\mathrm{BH}$ should be $\gtrsim 10^{52} \mathrm{erg}$. Observationally, the current upper limit for S-GRFs is $(7.8 \pm 1.0) \times 10^{51} \mathrm{erg}$, and the lower limit for BdHNe is $(2.44 \pm 0.22) \times 10^{52} \mathrm{erg}$ (see Ruffini et al. $2016 \mathrm{~b}$, for further details).

The above sub-classification is further supported by the fact that $\mathrm{GeV}$ emission, expected in presence of a rotating $\mathrm{BH}$, is indeed observed only in BdHNe (e.g. Ruffini et al. 2015a) and in S-GRBs (e.g. Aimuratov et al. 2017b; Ruffini et al. 2016a, 2015b; Muccino et al. 2013), and absent in XRFs and S-GRFs where no BH is formed (see Figure 10 and the Appendix in Ruffini et al. 2016b, for more details).

Therefore, the direct observation of the separatrix en- ergy between XRFs and BdHNe, as well as between SGRFs and S-GRBs, and their precise occurrence rates ratio, give crucial information on the actual NS critical mass value.

\section{INGREDIENTS SET-UP FOR THE COMPUTATION OF THE GW EMISSION AND ITS DETECTABILITY}

We have recalled in section 1 that the evolution of the binary progenitors of both short and long GRBs lead to compact binaries which will eventually merge in a characteristic timescale and emit GWs. We turn now in the following sections to assess the detectability of the GW emission by these merging binaries by Advanced LIGO.

In order to do this, we make the following drastic simplified assumptions:

1. Although it is manifest that the release of gravitational energy of the system in the merger phase is dominated by the $\mathrm{X}$, gamma-ray and $\mathrm{GeV}$ emission (see Table 1), we assume that the binary dynamics is only driven by the GW emission.

2. Consistent with the above GW emission dominance assumption, we further assume that the GW waveform is known and thus one can use the matched filtering technique to estimate the signalto-noise ratio. The actual $\mathrm{GW}$ waveform under the realistic conditions of electromagnetic emission dominance is still unknown.

3. To estimate the maximum distance of GW detectability we adopt optimally oriented sources with respect to the detector.

The above assumptions are made with the only aim of establishing an absolute upper limit to the GW emission and its putative detectability under the most optimistic conditions. Similarly, we assume that the binarity of the system does not compromise the interior structure of the NS (see Sec. 2).

The minimum GW frequency detectable by the broadband Advanced LIGO interferometer is $f_{\min }^{\mathrm{aLIGO}} \approx 10 \mathrm{~Hz}$ (LIGO Scientific Collaboration et al. 2015). Since during the binary inspiral the GW frequency is twice the orbital one, the above implies a binary is inside the Advanced LIGO band for orbital periods $P_{\text {orb }} \lesssim 0.2 \mathrm{~s}$.

\subsection{Systems to be analyzed}

The $\mathrm{CO}_{\text {core }} \mathrm{NS}$ binaries, in-states of XRFs and $\mathrm{BdHNe}$, and $\mathrm{CO}_{\text {core- }} \mathrm{BH}$ binaries, in-states of BH-SN, are not detectable by Advanced LIGO since they have orbital periods $P_{\text {orb }} \gtrsim 5$ min $\gg 0.2 \mathrm{~s}$ (Becerra et al. 2016). After their corresponding hypercritical accretion 
processes, it is clear that the out-states of both XRFs and BdHNe can become the in-states of short GRBs, as follows (Ruffini et al. 2016b; Fryer et al. 2015b; Becerra et al. 2015).

First let us discuss the out-states of XRFs. We have mentioned that XRFs can either get disrupted by the $\mathrm{SN}$ and lead to runaway NSs or, in the case the binary remains bound, lead to a $\nu$ NS-NS system. Since $\rho_{\mathrm{XRF}}>\rho_{\mathrm{S}-\mathrm{GRF}}+\rho_{\mathrm{S}-\mathrm{GRB}}$, such $\nu$ NS-NS binaries, outstates of XRFs, could be the in-states of S-GRFs (NSNS mergers leading to a MNS) and/or S-GRBs (NSNS mergers leading to a $\mathrm{BH}$ ). By denoting the total rate of short bursts as $\rho_{\text {short }} \equiv \rho_{\mathrm{S}-\mathrm{GRF}}+\rho_{\mathrm{S}-\mathrm{GRB}}$, our estimated rates would imply that the fraction of systems which appear to remain bound as $\nu$ NS-NS is $\left(\rho_{\text {short }} / \rho_{\mathrm{XRF}}\right) \approx 2 \%-8 \%$, while $92 \%-98 \%$ of XRFs are disrupted by the $\mathrm{SN}$ explosion. Interestingly, this is consistent with the fraction of bound NS-NS obtained in population synthesis analyses (see, e.g., Dominik et al. 2012; Postnov \& Yungelson 2014; Dominik et al. 2015; Fryer et al. 2015a; Belczynski et al. 2016, references therein and Appendix A.4 and A.5). Therefore, these merging $\nu$ NS-NS binaries are clearly included in the SGRF and S-GRB population. Such binaries are at birth undetectable by Advanced LIGO since they have initially $P_{\text {orb }} \gtrsim 5 \mathrm{~min} \gg 0.2 \mathrm{~s}$, but their merging can become detectable.

We have already recalled in the Introduction that in Fryer et al. (2015b) it was shown that, contrary to the case of XRFs, most BdHNe are expected to keep bound after the SN explosion in view of their short orbital periods and more massive accretion process. We have argued that those mergers would lead to the new class of short bursts, the U-GRBs (Fryer et al. 2015b), which however have still to be electromagnetically identified. The same applies to the $\nu \mathrm{NS}-\mathrm{BH}$ systems produced by BH-SN systems, with the only difference being the mass of the BH which, by definition of this subclass, can be larger than the NS critical mass since this $\mathrm{BH}$ is formed from direct collapse of a massive star. All the above merging $\nu \mathrm{NS}-\mathrm{BH}$ binaries are, by definition, the U-GRB population. Such binaries are at birth undetectable by Advanced LIGO because their initial orbital periods $P_{\text {orb }} \gtrsim 5 \mathrm{~min} \gg 0.2 \mathrm{~s}$, but their merger can become detectable.

In the case of NS-WD binaries, the WD large radius and its very likely tidal disruption by the NS make their GW emission hard to be detected (see, e.g., Paschalidis et al. 2009). Thus, we do not consider NS-WD binaries in the following GW discussion.

To summarize, we are going to analyze below the GW emission and detectability of S-GRF and S-GRB, the mergers of $\nu \mathrm{NS}-\mathrm{NS}$ produced by XRFs, as well as of UGRBs, which are the merger of the $\nu$ NS-BH produced by BdHNe and BH-SNe.

\subsection{Binary component masses}

For S-GRFs, we consider the simple case of nonspinning, equal-mass NS-NS merging binaries, i.e. $m_{1}=$ $m_{2}=m$. The precise value of the merging NS masses leading to a $\mathrm{BH}$ is still poorly known, thus we have chosen as an upper limit roughly half the maximum NS critical mass (see Sec. 2). Thus, we shall explore mass values $m \approx(1-1.7) M_{\odot}$.

For S-GRBs, we also consider non-spinning, equalmass NS-NS merging binaries. For self-consistency, we choose a range of component masses starting from the upper edge of the S-GRF one, i.e. $m \approx 1.7 M_{\odot}$, up to the maximum non-rotating stable mass, i.e. $m \approx$ $2.8 M_{\odot}$.

For U-GRBs, we adopt in the case of out-states of BdHNe, $m_{1}=1.5 M_{\odot}$ for the $\nu \mathrm{NS}$ and $m_{\mathrm{BH}}=2.7-$ $3.4 M_{\odot}$ for the $\mathrm{BH}$ (see Sec. 2). In the case of out-states of BH-SNe, we adopt $m_{1}=1.5 M_{\odot}$ for the $\nu \mathrm{NS}$ and $m_{\mathrm{BH}}=3.4-10 M_{\odot}$ for the $\mathrm{BH}$ consistent with the assumption that the $\mathrm{BH}$ in this subclass has been previously formed in the binary evolution and therefore it can have a mass larger than the NS critical mass.

\subsection{Signal-to-noise ratio}

We first recall the main ingredients needed to estimate the detectability of the aforementioned merging binaries associated with the different GRB classes. The signal $h(t)$ induced in the detector is:

$$
h(t)=F_{+}(\theta, \phi, \psi) h_{+}(t, \iota, \beta)+F_{\times}(\theta, \phi, \psi) h_{\times}(t, \iota, \beta),
$$

where $h_{+}$and $h_{\times}$are the two polarizations of the GW; $\iota$ and $\beta$ are the polar and azimuthal angles of the unit vector from the source to the detector, relative to a coordinate system centered in the source. The detector pattern functions $F_{+}$and $F_{\times}$depend on the localization of the source with respect to the detector, i.e. they depend on the spherical polar angles $\theta$ and $\phi$ of the source relative to a coordinate system centered in the detector. The pattern functions also depend on the polarization angle $\psi$.

Since the GW signal might be deep inside the noise, the signal-to-noise ratio, denoted hereafter by $\rho$, is usually computed using the matched filter technique, i.e. (Flanagan \& Hughes 1998):

$$
\rho^{2}=4 \int_{0}^{\infty} \frac{|\tilde{h}(f)|^{2}}{S_{n}(f)} d f,
$$

where $f$ is the GW frequency in the detector's frame, $\tilde{h}(f)$ is the Fourier transform of $h(t)$ and $\sqrt{S_{n}(f)}$ is the one-sided amplitude spectral density (ASD) of the Advanced LIGO noise. We recall that in the detector's 
frame the GW frequency is redshifted by a factor $1+z$ with respect to the one in the source's frame, $f_{s}$, i.e. $f=f_{s} /(1+z)$.

The exact position of the binary relative to the detector and the orientation of the binary rotation plane are usually unknown, thus it is a common practice to estimate the signal-to-noise ratio averaging over all the possible locations and orientations, i.e.:

$$
\left\langle\rho^{2}\right\rangle=4 \int_{0}^{\infty} \frac{\left\langle|\tilde{h}(f)|^{2}\right\rangle}{S_{n}(f)} d f=4 \int_{0}^{\infty} \frac{h_{c}^{2}(f)}{f^{2} S_{n}(f)} d f,
$$

with $h_{c}(f)$ the characteristic strain (Flanagan \& Hughes 1998)

$$
h_{c}=\frac{(1+z)}{\pi d_{l}} \sqrt{\frac{\left\langle F_{+}^{2}\right\rangle}{2} \frac{G}{c^{3}} \frac{d E}{d f_{s}}[(1+z) f]},
$$

where

$$
d_{l}=\frac{(1+z) c}{H_{0}} \int_{0}^{z}\left[\Omega_{M}(1+x)^{3}+\Omega_{\Lambda}\right]^{-1 / 2} d x
$$

is the source luminosity distance and we have used the fact that $\left\langle F_{+}^{2}\right\rangle=\left\langle F_{\times}^{2}\right\rangle$ and $\left\langle F_{+} F_{\times}\right\rangle=0$. We recall that $\left\langle F_{+}^{2}\right\rangle=1 / 5$ for an interferometer and $\left\langle F_{+}^{2}\right\rangle=4 / 15$ for a resonant bar (see, e.g., Maggiore 2007). We adopt a $\Lambda$ CDM cosmology with $H_{0}=71 \mathrm{~km} \mathrm{~s}^{-1} \mathrm{Mpc}^{-1}$, $\Omega_{M}=0.27$ and $\Omega_{\Lambda}=0.73$ (Rigault et al. 2015). It is important to recall that, as we have mentioned, we are interested in estimating the GW detectability under the most optimistic conditions. Thus, to estimate the maximum distance of GW detectability we adopt in Sec. 3 the ansatz of optimally oriented sources with respect to the detector. The above averaging procedure is here used with the only aim of giving an estimate of the GW strain amplitude, $h_{c}$, compared and contrasted below in Sec. 5 with the detectors strain-noise.

\section{GW ENERGY SPECTRUM}

In general, a GW-driven binary system evolves in time through two regimes: the first is the inspiral regime and the second, which we refer hereafter to as merger regime, is composed in the most general case of the final plunge, of the merger, and of the ringdown (oscillations) of the newly formed object.

\subsection{Inspiral regime}

During the inspiral regime the system evolves describing quasi-circular orbits and it is well described by the traditional point-like quadrupole approximation (Peters \& Mathews 1963; Peters 1964; Rees et al. 1974; Landau \& Lifshitz 1975). The GW frequency is twice the orbital frequency $\left(f_{s}=2 f_{\text {orb }}\right)$ and grows monotonically. The energy spectrum during the inspiral regime is:

$$
\frac{d E}{d f_{s}}=\frac{1}{3}(\pi G)^{2 / 3} M_{c}^{5 / 3} f_{s}^{-1 / 3},
$$

where $M_{c}=\mu^{3 / 5} M^{2 / 5}=\nu^{3 / 5} M$ is the called chirp mass, $M=m_{1}+m_{2}$ is the total binary mass, $\mu=$ $m_{1} m_{2} / M$ is the reduced mass, and $\nu \equiv \mu / M$ is the symmetric mass-ratio parameter. A symmetric binary $\left(m_{1}=m_{2}\right)$ corresponds to $\nu=1 / 4$ and the test-particle limit is $\nu \rightarrow 0$. The total energy emitted during this regime can be estimated as the difference of the energy of the binary between infinity and the one at the last circular orbit (LCO). For a test-particle in the Schwarzschild background the $\mathrm{LCO}$ is located at $r_{\mathrm{LCO}}=6 \mathrm{GM} / \mathrm{c}^{2}$, its energy is $\sqrt{8 / 9} \mu c^{2}$, then:

$$
\Delta E_{\text {insp }}=(1-\sqrt{8 / 9}) \mu c^{2} .
$$

\subsection{Merger regime}

The GW spectrum of the merger regime is characterized by a GW burst (see, e.g., Davis et al. 1971; Shibata \& Taniguchi 2011; Bernuzzi et al. 2015). Thus, to estimate whether this part of the signal contributes to the signal-to-noise ratio, it is sufficient to estimate the location of the GW burst in the frequency domain and its energy content. We recall that, in general, the merger regime is composed of plunge+merger+ringdown. The frequency range spanned by the $\mathrm{GW}$ burst is $\Delta f=$ $f_{\text {qnm }}-f_{\text {merger }}$, where $f_{\text {merger }}$ is the frequency at which the merger starts and $f_{\mathrm{qnm}}$ is the frequency of the ringing modes of the newly formed object after the merger, and the energy emitted is $\Delta E_{\text {merger }}$. With these quantities defined, we can estimate the typical value of the merger regime spectrum as:

$$
\left(\frac{d E}{d f_{s}}\right)_{\text {merger }} \sim \frac{\Delta E_{\text {merger }}}{\Delta f} .
$$

Numerical relativity simulations (e.g. Shibata \& Taniguchi 2011; Bernuzzi et al. 2015) show that finite size effects might end the inspiral regime before the LCO. After this point, the GW spectrum damps exponentially. For the case of NS-NS the merger starts in an orbit larger than the LCO, and for the case of a NS-BH, as we will see below, the merger can occur below the LCO making the spectrum similar to a $\mathrm{BH}-\mathrm{BH}$ merger. When the merger occurs well before the LCO, there is no plunge. Therefore, the emitted energy will be less than the case when the plunge is present. We can therefore obtain an upper limit to $\Delta E_{\text {merger }}$ by adopting the energy emitted during the plunge-merger-ringdown of a BH-BH merger (Detweiler \& Szedenits 1979)

$$
\Delta E_{\text {merger }} \approx 0.5 \nu^{2} M c^{2} .
$$

To complete the estimate of the merger regime spectrum, we have to estimate the value of $\Delta f$ in the different cases of interest. 
Table 3. Properties of the GW emission of S-GRFs, S-GRBs and U-GRBs. We have made the drastic simplified assumption that the binary evolution is only driven by GW emission, although it is manifest that the gravitational energy of the system in the merger phase is dominated by the radio, optical, X, gamma-ray and GeV emission (see Table 1). This assumption is made with the only aim of establishing an absolute upper limit to the GW emission and its detectability under the most optimistic conditions. Column 1: GRB subclass. Column 2: energy emitted in GWs during the inspiral regime $\Delta E_{\text {insp }}$ given by Eq. (7).

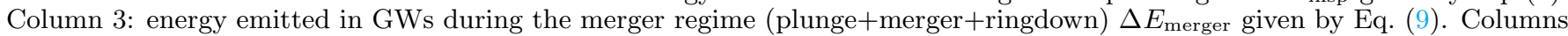
4: GW frequency at merger. Column 5: GW frequency of the ringdown regime. Column 6: lowest cosmological redshift value $z_{\text {min }}^{\text {obs }}$ at which each subclass has been observed. Column 7: luminosity distance corresponding to $z_{\text {min }}^{\text {obs }}, d_{l_{\text {min }}}$, estimated from Eq. (5). Columns 8-10: GW horizon calculated with the sensitivity of Advanced LIGO during the O1 and O2 runs and with the expected final sensitivity including LIGO-India $(2022+)$, respectively. It can be seen that the current GW horizon is much smaller than the observed distances of GRBs, impeding a positive detection by Advanced LIGO. Only in the case of U-GRB (BH-SN) it is foreseen a possible detection during the run 2022+. See also Table 4. We have used for S-GRFs $(1.4+1.4) M_{\odot}$, for S-GRBs $(2.0+2.0) M_{\odot}$ and, for U-GRBs $(1.5+3.0) M_{\odot}$ and $(1.5+10.0) M_{\odot}$ respectively for the out-states of BdHNe and of BH-SN. Even if no U-GRB has yet been identified, we use here the values of $z_{\text {min }}^{\text {obs }}$ and $d_{l_{\min }}$ corresponding to the closest BdHN observed.

\begin{tabular}{lccccccccc}
\hline \hline & $\begin{array}{c}\Delta E_{\text {insp }} \\
(\mathrm{erg})\end{array}$ & $\begin{array}{c}\Delta E_{\text {merger }} \\
(\mathrm{erg})\end{array}$ & $\begin{array}{c}f_{\text {merger }} \\
(\mathrm{kHz})\end{array}$ & $\begin{array}{c}f_{\mathrm{qnm}} \\
(\mathrm{kHz})\end{array}$ & $z_{\text {min }}^{\text {obs }}$ & $\begin{array}{c}d_{l_{\text {min }}} \\
(\mathrm{Mpc})\end{array}$ & \multicolumn{2}{c}{$d_{\mathrm{GW}}(\mathrm{Mpc})$} & $\mathrm{O} 2$ \\
\hline S-GRF & $7.17 \times 10^{52}$ & $1.60 \times 10^{53}$ & 1.20 & 3.84 & 0.111 & 508.70 & $90.51-181.02$ & $181.02-271.52$ & 452.54 \\
S-GRB & $1.02 \times 10^{53}$ & $2.28 \times 10^{53}$ & 1.43 & 2.59 & 0.903 & 5841.80 & $121.84-243.67$ & $243.67-365.51$ & 609.18 \\
U-GRB & $1.02 \times 10^{53}$ & $2.03 \times 10^{52}$ & 0.98 & 2.30 & 0.169 & 804.57 & $126.71-253.43$ & $253.43-380.14$ & 633.57 \\
U-GRB (BH-SN) & $1.34 \times 10^{53}$ & $1.35 \times 10^{53}$ & 0.38 & 0.90 & 0.169 & 804.57 & $197.86-395.71$ & $395.71-593.57$ & 989.28 \\
\hline \hline
\end{tabular}

\subsubsection{NS-NS merger}

The approach to the merger point, $r=r_{\text {merger }}$, depends on the nature of the binary system. Typically, the merger is assumed to start at the point of maximum GW strain (see, e.g., Bernuzzi et al. 2015, and references therein). However, since the transition from a binary system to a single merged object is not sharply definable, there can be found different definitions of the merger point in the literature (see, e.g., Kawaguchi et al. 2015). For our purpose it is sufficient to estimate the frequency at "contact", namely the frequency at a binary separation $r_{\text {contact }} \approx r_{1}+r_{2}$ where $r_{i}$ is the radius of the $i$-component. This certainly sets a lower limit to the frequency at maximum strain at merger, i.e. $r_{\text {contact }} \gtrsim r_{\text {merger }}$. Thus, we adopt for these systems:

$$
f_{\text {merger }}^{\mathrm{NS}-\mathrm{NS}} \approx f_{\text {contact }}^{\mathrm{NS}-\mathrm{NS}}=\frac{1}{\pi} \frac{c^{3}}{G M}\left[\frac{\mathcal{C}_{1} \mathcal{C}_{2}(1+q)}{\mathcal{C}_{1}+q \mathcal{C}_{2}}\right]^{3 / 2},
$$

where $q=m_{2} / m_{1}$ is the mass-ratio which is related to the symmetric mass-ratio parameter by $\nu=q /(1+$ $q)^{2}$, and $\mathcal{C}_{i} \equiv G m_{i} / c^{2} r_{i}$ is the compactness of the $i$ component.

For a mass-symmetric NS-NS binary, we have that $f_{\text {contact }}^{\mathrm{NS}-\mathrm{NS}} \approx(1 / \pi)\left(c^{3} / G\right) \mathcal{C}_{\mathrm{NS}}^{3 / 2} / M$, where $\mathcal{C}_{\mathrm{NS}} \equiv \mathcal{C}_{1}=\mathcal{C}_{2}$ is the compactness parameter of the initial NS. For example, for the NL3 EOS, the NS compactness lies in the range $\mathcal{C}_{\mathrm{NS}} \approx 0.14-0.3$ for a NS mass $1.4-2.8 M_{\odot}$ (see, e.g., Cipolletta et al. 2015). Thus, using the same EOS we have, for an $M=(1.4+1.4) M_{\odot}=2.8 M_{\odot}$ binary, $f_{\text {contact }}^{\mathrm{NS}-\mathrm{NS}} \approx 1.34 \mathrm{kHz}$, and for an $M=(2.0+2.0) M_{\odot}=$ $4.0 M_{\odot}$ binary, $f_{\text {contact }}^{\mathrm{NS}-\mathrm{NS}} \approx 1.43 \mathrm{kHz}$.

In the merger regime either a $\mathrm{BH}$ or a $\mathrm{MNS}$ can be formed. If the merger does not lead to a $\mathrm{BH}$, the merger frequency is dominated by the frequency of the quasinormal modes of the MNS formed. This frequency is of the order of

$$
f_{\mathrm{qnm}}^{\mathrm{MNS}} \approx \frac{1}{\pi}\left(\frac{G M}{R^{3}}\right)^{1 / 2}=\frac{1}{\pi}\left(\frac{c^{3}}{G}\right) \frac{\mathcal{C}_{\mathrm{MNS}}^{3 / 2}}{M},
$$

where $R$ is the radius of the MNS and $\mathcal{C}_{\mathrm{MNS}} \equiv$ $G M /\left(c^{2} R\right)$ is its compactness. Thus, in the case of SGRFs the value of $\Delta f$ is

$$
\begin{aligned}
\Delta f_{\mathrm{S}-\mathrm{GRF}} & \equiv f_{\mathrm{qnm}}^{\mathrm{MNS}}-f_{\text {contact }}^{\mathrm{NS}-\mathrm{NS}} \\
& \approx\left(\mathcal{C}_{\mathrm{MNS}}^{3 / 2}-\mathcal{C}_{\mathrm{NS}}^{3 / 2}\right) \frac{c^{3}}{\pi G M} .
\end{aligned}
$$

If the merger forms a $\mathrm{BH}$, the merger frequency is dominated by the frequency of the quasi-normal modes of the $\mathrm{BH}$ formed, namely the GW-burst spectrum peaks at the frequency (Davis et al. 1971, 1972)

$$
f_{\mathrm{qnm}}^{\mathrm{BH}} \approx \frac{0.32}{2 \pi} \frac{c^{3}}{G M},
$$

i.e. $f_{\mathrm{qnm}} \approx 3.4 \mathrm{kHz}$ for a Schwarzschild $\mathrm{BH}$ of $3 M_{\odot}$. In the case of a rotating $\mathrm{BH}$, namely a Kerr $\mathrm{BH}$, the peak frequency shifts to higher values (Detweiler 1980). Thus, the value of $f_{\mathrm{qnm}}^{\mathrm{BH}}$ given by Eq. (13) can be considered as a lower bound to the actual peak frequency. Thus, in the case of S-GRBs the value of $\Delta f$ is

$$
\begin{aligned}
\Delta f_{\mathrm{S}-\mathrm{GRB}} & \equiv f_{\mathrm{qnm}}^{\mathrm{BH}}-f_{\text {contact }}^{\mathrm{NS}-\mathrm{NS}} \\
& \approx\left(0.16-\mathcal{C}_{\mathrm{NS}}^{3 / 2}\right) \frac{c^{3}}{\pi G M} .
\end{aligned}
$$

In either case of $\mathrm{BH}$ or MNS formation, it is satisfied $f_{\text {qnm }}>f_{\text {contact }}$. It can be checked that the above frequency estimates are consistent with values obtained 
from full numerical relativity simulations (see, e.g., Anninos et al. 1995; Bernuzzi et al. 2015).

\subsubsection{NS-BH merger}

For a NS-BH merger, the approach to merger is different since general relativistic effects avoid the objects to go all the way to the "contact" point following circular orbits. For example, let us assume $m_{1}=$ $m_{\mathrm{BH}} \approx 3 M_{\odot}$ and $m_{2}=M_{\mathrm{NS}} \approx 1.5 M_{\odot}$, so $M=$ $1.5+3.0 M_{\odot}=4.5 M_{\odot}$. In this case $r_{1}=2 G m_{\mathrm{BH}} / c^{2}$ (for a Schwarzschild $\mathrm{BH}$ ) and $r_{2}=G m_{2} /\left(c^{2} \mathcal{C}_{2}\right)$, so $r_{\text {contact }} \approx 3.3 G M / c^{2}$. Within the test-particle limit, the LCO around a Schwarzschild $\mathrm{BH}$ occurs at $r_{\mathrm{LCO}}=$ $6 G m_{\mathrm{BH}} / c^{2} \approx 6 G M / c^{2}>r_{\text {contact }}$. Thus, we have that $r_{\text {contact }}<r_{\mathrm{LCO}}$ which suggests that a NS-BH binary, similar to the case of a $\mathrm{BH}-\mathrm{BH}$ one, can pass from the inspiral regime, to the plunge from $r_{\text {plunge }}=r_{\mathrm{LCO}}$ to merger at $r_{\text {merger }} \approx r_{\text {contact }}$, to the ringing of the newly formed BH. At $r_{\text {plunge }}$, the GW frequency is

$$
f_{\text {plunge }}^{\mathrm{NS}-\mathrm{BH}} \approx \frac{1}{\pi}\left(\frac{G M}{r_{\mathrm{LCO}}^{3}}\right)^{1 / 2}=\frac{1}{\pi 6^{3 / 2}}\left(\frac{c^{3}}{G M}\right),
$$

and as in the previous case of $\mathrm{BH}$ formation from a NSNS merger, the NS-BH post-merger GW spectrum will be dominated by frequencies given by Eq. (13). Namely, for the present example $f_{\text {plunge }}^{\mathrm{NS}-\mathrm{BH}} \approx 980 \mathrm{~Hz}$ and $f_{\mathrm{qnm}}^{\mathrm{BH}} \approx$ $2.3 \mathrm{kHz}$.

Thus, in the case of NS-BH merger (U-GRB subclass), the value of $\Delta f$ is

$$
\Delta f_{\mathrm{U}-\mathrm{GRB}} \equiv f_{\mathrm{qnm}}^{\mathrm{BH}}-f_{\text {plunge }}^{\mathrm{NS}-\mathrm{BH}} \approx 0.092 \frac{c^{3}}{\pi G M} .
$$

In the above analysis we have neglected the possibility that the NS can be tidally disrupted by the BH before it reaches $r=r_{\mathrm{LCO}}$. The NS is disrupted by the $\mathrm{BH}$ if $r_{\mathrm{LCO}}<r_{\mathrm{td}}$, where $r_{\mathrm{td}}$ is the tidal disruption radius. The value of $r_{\mathrm{LCO}}$ and $r_{\mathrm{td}}$ for a NS-BH system depends both on the binary mass-ratio $q \equiv m_{2} / m_{1} \leq 1$ and on the NS compactness $\mathcal{C}_{\mathrm{NS}}$ which depends, in turn, on the NS mass and EOS. Numerical simulations of NS-BH binary mergers adopting a polytropic EOS for the NS matter suggest $r_{\mathrm{td}} \approx 2.4 q^{-1 / 3} R_{\mathrm{NS}}$ and $r_{\mathrm{LCO}} \approx 6 G M / c^{2}\left[1-0.44 q^{1 / 4}\left(1-3.54 \mathcal{C}_{\mathrm{NS}}\right]^{-2 / 3}\right.$ (see Shibata \& Taniguchi 2011, and references therein). The ratio $r_{\mathrm{td}} / r_{\mathrm{LCO}}$ is a decreasing function of the $\mathrm{BH}$ mass for given NS mass (but always close to unity). If we extrapolate these results to $\mathrm{BH}$ masses in the range (310) $M_{\odot}$ and a NS of $1.5 M_{\odot}$ obeying the NL3 EOS we have $r_{\mathrm{LCO}}<r_{\mathrm{td}}$ for $m_{\mathrm{BH}} \lesssim 6 M_{\odot}$ and $r_{\mathrm{LCO}}>r_{\mathrm{td}}$ otherwise. It is clear that the specific range of NS and $\mathrm{BH}$ masses for which there is tidal disruption is highly sensitive to the compactness of the NS, hence to the nuclear EOS, and thus more simulations using a wide set of updated nuclear EOS is needed to assess this issue. If tidal disruption occurs, the inspiral regime will cut-off at a GW frequency

$$
f_{\mathrm{td}}^{\mathrm{NS}-\mathrm{BH}} \approx \frac{1}{\pi}\left(\frac{G M}{r_{\mathrm{td}}^{3}}\right)^{1 / 2} .
$$

Since $r_{\mathrm{td}}$ is near $r_{\mathrm{LCO}}$ for our systems, and to not introduce further uncertainties in our estimates, we shall adopt that the inspiral regime of our NS-BH systems ends at the GW frequency given by Eq. (15).

\section{CHARACTERISTIC STRAIN AND DETECTORS SENSITIVITY}

From Eqs. (6) and (8) and with the knowledge of the energy released in GWs (9) and the spanned frequencies in the merger regime (see Table 3), we can estimate the characteristic strain (4) which can be compared and contrasted with the strain noise of GW detectors.

Fig. 1 shows the GW signal ASD produced by SGRFs, S-GRBs and U-GRBs, obtained with the aid of Eq. (4). In this figure we adopt: a $(1.4+1.4) M_{\odot}$ $\nu$ NS-NS merger for S-GRFs, a $(2.0+2.0) M_{\odot} \nu$ NS-NS merger for S-GRBs, a $(1.5+3.0) M_{\odot} \nu \mathrm{NS}-\mathrm{BH}$ merger for U-GRBs produced by out-states of BdHNe, and a $(1.5+10.0) M_{\odot} \nu \mathrm{NS}-\mathrm{BH}$ merger for U-GRBs produced by out-states of BH-SNe. We have assumed in this plot that these sources are located at the closest luminosity distance $d_{l}$ at which each subclass has been observed (see Table 3 for details). We show the noise ASD of Advanced LIGO in the current run (O1) and in the expected 2022+ run (Abbott et al. 2016); the expected noise ASD of Advanced Virgo (BNS-optimized; Abbott et al. 2016); the expected noise ASD of the space-based interferometer eLISA for the N2A1, N2A2 and N2A5 configurations (see, e.g., Klein et al. 2016); and the noise ASD of the NAUTILUS bar detector for a $1 \mathrm{~ms}$ GW burst (Astone et al. 2006, 2008). Narrow-band resonant bar detectors (such as ALLEGRO, AURIGA, EXPLORER, NAUTILUS and NIOBE) are sensitive within a bandwidth of $\sim 1-10 \mathrm{~Hz}$ around the resonant frequency which is typically $f_{0} \sim 1 \mathrm{kHz}$ (see, e.g., Table 2 in Camp \& Cornish 2004, for a summary of the properties of the bar detectors). The bar detector with the wider bandwidth is NAUTILUS with a minimum strain spectral noise $\sqrt{S_{n}}=10^{-21} \mathrm{~Hz}^{-1 / 2}$ at $f_{0}=935 \mathrm{~Hz}$ and $\sqrt{S_{n}} \leq 10^{-20} \mathrm{~Hz}^{-1 / 2}$ in a bandwidth $\sim 30 \mathrm{~Hz}$ around $f_{0}$ (Astone et al. 2008). This implies that a $1 \mathrm{~ms} \mathrm{GW}$ burst would be detected by this instrument if it has a strain amplitude $h \gtrsim 3 \times 10^{-19}$ (Astone et al. 2006, 2008).

From this figure we can conclude for the NS-NS and NS-BH binaries associated with S-GRFs, S-GRBs and U-GRBs:

1. Before merging: they transit, during their inspiral regime which spans the frequency range 


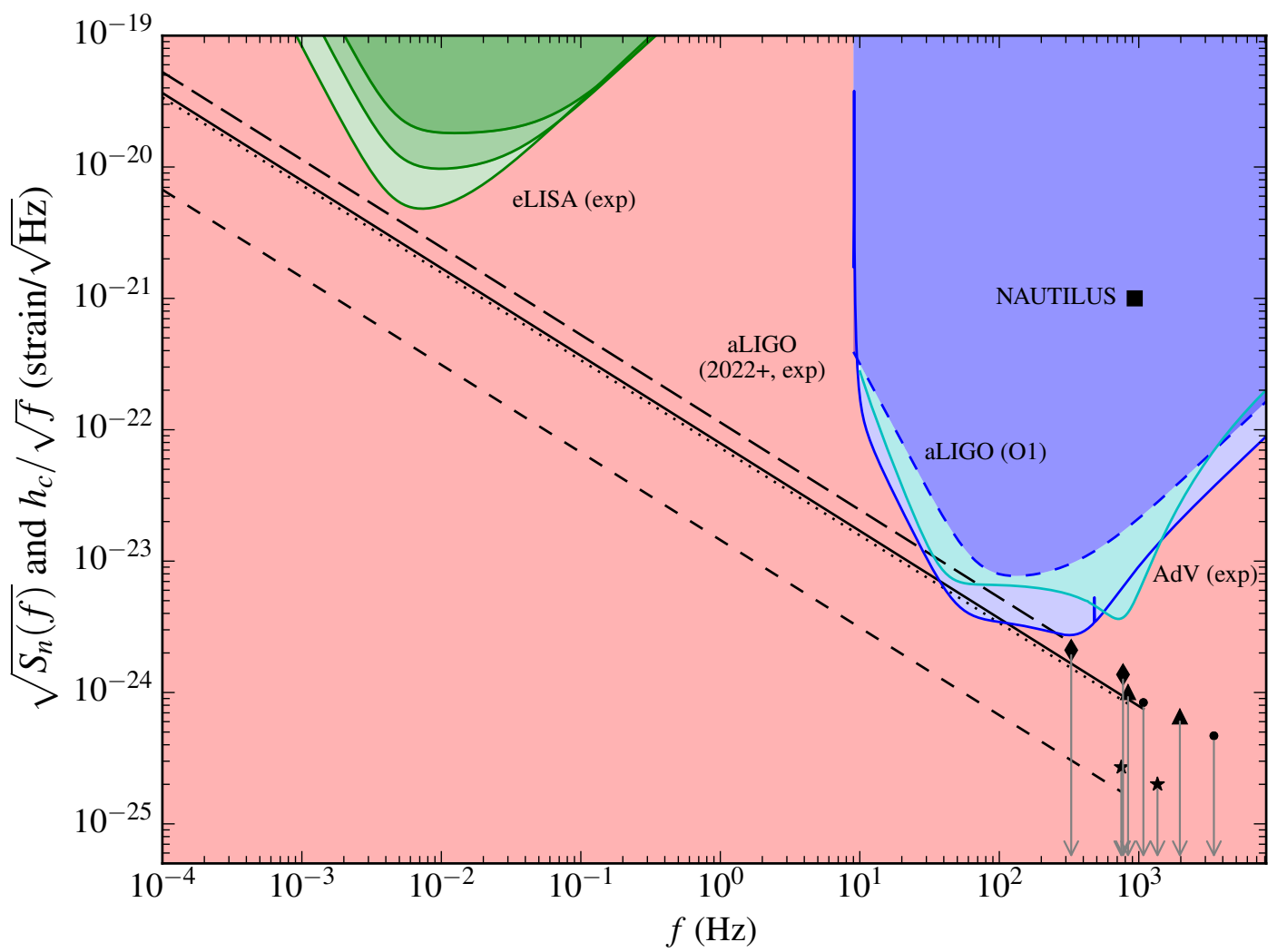

Figure 1. Comparison of the signal's ASD $h_{c} / \sqrt{f}$ of S-GRFs, S-GRBs and U-GRBs with the noise's ASD $\sqrt{S_{n}(f)}$, where $S_{n}$ is the power spectrum density of the detector's noise of eLISA, of Advanced LIGO (aLIGO) and of the bar detector NAUTILUS. The red lines, from top to bottom, are the expected noise's ASD of the N2A1, N2A2 and N2A5 configurations of eLISA (Klein et al. 2016). The dashed and continuous red lines correspond to the noise's ASD respectively of Advanced LIGO O1 run (2015/2016) and of the expected Advanced LIGO 2022+ run (Abbott et al. 2016), and the cyan line is the expected noise's ASD of Advanced Virgo (AdV) BNS-optimized (Abbott et al. 2016). The filled square indicates the noise's ASD of the NAUTILUS resonant bar for a $1 \mathrm{~ms}$ GW burst (Astone et al. 2006, 2008). The red filled area indicates the region of undetectability by any of the above instruments. We recall that in this plot the GW frequency is redshifted by a factor $1+z$ with respect to the source frame value, i.e. $f=f_{s} /(1+z)$, for which we use the cosmological redshift and corresponding luminosity distance of the closest observed source of each subclass (see Table 3). The following three curves correspond to the inspiral regime of the coalescence: S-GRFs with $(1.4+1.4) M_{\odot}$ (solid curve), S-GRBs with $(2.0+2.0) M_{\odot}$ (short-dashed curve), U-GRB with $(1.5+3.0) M_{\odot}$ (dotted curve) from out-states of BdHNe, and U-GRB with $(1.5+10.0) M_{\odot}$ (long dashed curve) from out-states of BH-SNe. The dot, star, triangle and diamond correspond to $h_{c}$ in the merger regime for S-GRFs, S-GRBs, U-GRBs from out-states of BdHNe, and U-GRBs from out-states of BH-SNe, respectively. The first point is located at $f_{\text {merger }} /(1+z)$ and the second at $f_{\mathrm{qnm}} /(1+z)$ (see Table 3). The down-arrows indicate that these estimates have to be considered as upper limits since we have assumed that all the energy release in the system goes in GWs, which clearly overestimates the GW energy output in view of the dominance of the electromagnetic emission (see Table 4). We have also overestimated the GW energy in the merger regime by using Eq. (9) which is the expected GW energy emitted in the plunge+merger+ringdown phases of a BH-BH merger. For binary mergers involving NSs, as we have discussed in Sec. 4, the energy released in GWs must be necessarily lower than this value.

$f<f_{\text {merger }} /(1+z)$ (see in Table 3 the frequencies and redshift), first the eLISA frequency band to then enter the Advanced LIGO-Virgo ones in the final orbits prior the merging process (when $P_{\text {orb }}<0.2 \mathrm{~s}$ ). The narrow bandwidth of the bar detectors does not cover these frequencies. For the adopted distances we see that the characteristic strain generated by all these sources is below the sensitivity of eLISA. S-GRFs are below the sensitivity of Advanced LIGO (O1), Advanced Virgo and NAUTILUS, but inside the sensitivity of Advanced LIGO $(2022+)$. S-GRBs are below the sen- sitivity of Advanced LIGO (all runs), Advanced Virgo and NAUTILUS. U-GRBs from out-states of BdHNe are below the sensitivity of Advanced LIGO (O1), Advanced Virgo and NAUTILUS, but inside the sensitivity of Advanced LIGO (2022+). U-GRBs from out-states of BH-SNe are below the sensitivity of Advanced LIGO (O1) and NAUTILUS, inside the sensitivity of Advanced LIGO $(2022+)$, and marginally inside the sensitivity of Advanced Virgo.

2. Merging: the merging regime, which expands frequencies from $f_{\text {contact }} /(1+z)$ to $f_{\mathrm{qnm}} /(1+z)$ (see 
in Table 3 the frequencies and redshift), is outside the eLISA frequency band but inside the Advanced LIGO-Virgo and bar detectors ones. The characteristic strain in this final merger phase $h \sim 10^{-24}-10^{-23}$ is, unfortunately, well below the sensitivity of all of them (see, also, Kobayashi \& Mészáros 2003, for similar conclusions for Advanced LIGO).

From the above it can be seen that the most interesting instrument for the possible detection of the GW emission from binaries associated with GRBs is Advanced LIGO. Therefore, we estimate in the next section the expected detection rates by Advanced LIGO-Virgo (see Fig. 2 and Table 4).

\section{GW DETECTION RATE}

We assume a threshold for the Advanced LIGO-Virgo single detector $\rho_{0}=8$ (Abbott et al. 2016). This minimum $\rho_{0}$ defines a maximum detection distance or GW horizon distance, which is denoted as $d_{\mathrm{GW}}$. This horizon corresponds to the most optimistic case when the binary is just above the detector and the binary plane is parallel to the detector plane, i.e. $\theta=\phi=\iota=0$ (Allen et al. 2012):

$$
d_{\mathrm{GW}}=\frac{2 A}{\rho_{0}}\left(\int_{0}^{\infty} \frac{f^{-7 / 3}}{S_{n}(f)} d f\right)^{1 / 2},
$$

where $A=5 /\left(24 \pi^{4 / 3}\right)^{1 / 2}\left(G M_{c} / c^{3}\right)^{5 / 6} c$. Since not all the sources are optimally aligned with the detector, the number of detected sources inside a sphere of radius $d_{\mathrm{GW}}$ will be a fraction $\mathcal{F}^{3}$ of the total. This fraction determines the so-called "range" of the detector, $\mathcal{R}=\mathcal{F} d_{\mathrm{GW}}$, where $\mathcal{F}^{-1}=2.2627$ (see Finn \& Chernoff 1993, for details). In order to give an estimate of the annual number of detectable binaries associated with GRBs we use the search volume as computed in (Abbott et al. 2016), $\mathcal{V}_{s}=V_{\max }^{\mathrm{GW}} \mathcal{T}$, where $V_{\max }^{\mathrm{GW}}=(4 \pi / 3) \mathcal{R}^{3}$ and $\mathcal{T}$ is the observing time accounting for the detectors duty cycles. We use here the lower and upper values of $\mathcal{R}$ and $\mathcal{V}_{s}$ for a $(1.4+1.4) M_{\odot}$ NS binary for the different observational campaigns reported in (Abbott et al. 2016): 2015/2016 (O1) with $\mathcal{R}=40-80 \mathrm{Mpc}, \mathcal{T}=3$ months, $\mathcal{V}_{S}=\left(0.5^{-}\right.$ 4) $\times 10^{5} \mathrm{Mpc}^{3} \mathrm{yr} ; 2016 / 2017(\mathrm{O} 2)$ with $\mathcal{R}=80-120 \mathrm{Mpc}$, $\mathcal{T}=6$ months, $\mathcal{V}_{S}=(0.6-2) \times 10^{6} \mathrm{Mpc}^{3}$ yr; $2017 / 2018$ (O3) with $\mathcal{R}=120-170 \mathrm{Mpc}, \mathcal{T}=9$ months, $\mathcal{V}_{S}=(3-$ 10) $\times 10^{6} \mathrm{Mpc}^{3} \mathrm{yr}$, and the one by the entire network including LIGO-India at design sensitivity $(2022+)$ with $\mathcal{R}=200 \mathrm{Mpc}, \mathcal{T}=1 \mathrm{yr}, \mathcal{V}_{S}=4 \times 10^{7} \mathrm{Mpc}^{3}$ yr. We can use the above information for a $(1.4+1.4) M_{\odot}$ binary and extrapolate it to other binaries with different masses using the property that $d_{\mathrm{GW}}$ scales with the chirp mass as $M_{c}^{5 / 6}$ (see Eq. 18). We show in Table 3 GW horizon for a specific value of the binary component masses expected for S-GRFs, S-GRBs and U-GRBs (see section 3.2).

From the inferred occurrence rates $\rho_{\mathrm{GRB}}$ (not to be confused with signal-to-noise ratio $\rho$ ) summarized in Table 1, we show in Fig. 2 the expected number of GW detections by Advanced LIGO-Virgo for each observational campaign

$$
\dot{N}_{\mathrm{GW}}=\rho_{\mathrm{GRB}} \mathcal{V}_{s}
$$

for S-GRFs, S-GRBs, and U-GRBs as a function of the binary component masses (see section 3.2).

We compare and contrast in Table 4 for the GRB subclasses: the expected GW detection rate by Advanced LIGO-Virgo given by Eq. (19), $\dot{N}_{\mathrm{GW}}$, the inferred occurrence rate of GRBs, $\dot{N}_{\mathrm{GRB}}$, and the observed GRB rate from $\gamma$-ray telescopes (AGILE, BATSE, BeppoSAX, Fermi, HETE-II, INTEGRAL, Konus/WIND and Swift), simply estimated as $\dot{N}_{\mathrm{GRB}}^{\mathrm{obs}}=N_{\mathrm{GRB}}^{\mathrm{obs}} / T_{\text {obs }}$ where $N_{\mathrm{GRB}}^{\mathrm{obs}}$ is the number of GRBs detected in the observing time $T_{\text {obs }}$. The rate $\dot{N}_{\mathrm{GRB}}$ is obtained from the GRB specific rate through the reconstruction of the GRB luminosity function and the study of its evolution with the redshift (see Ruffini et al. 2016b, and Appendix B for details). This estimate, therefore, is larger than $\dot{N}_{\text {GRB }}^{\text {obs }}$ which is limited to those events beyond the detector sensitivity threshold, falling inside its field of view and within its operational time.

\section{CONCLUSIONS}

Short and long GRBs have been divided into 7 subclasses according to their binary nature (Ruffini et al. 2016b). We summarize in Table 1 their main physical properties characterizing the outcome of X-rays, gamma-rays, high-energy and ultra high-energy detectors, as well as their occurrence rate. Particularly important for the present work is the specification of the in-states and out-states of the GRB progenitors.

With the knowledge of the nature of the compact object binaries associated with each GRB subclass, and the relevance of the NS structure and critical mass in Sec. 2, we introduce in Sec. 3 the main ingredients for the computation of the GW emission and detectability for such systems. We describe in Sec. 4 the general properties of the GW emission during the inspiral and merger regimes of these binaries. We argue that S-GRFs, S-GRBs and U-GRBs are the GRB subclasses relevant for the GW analysis. It is manifest that the release of the gravitational energy of the system in the merger phase is dominated by the X-rays, gamma-rays and GeV emission (see Table 1). In order to evaluate the GW emission we have made in this work the drastic simplified assumption that the binary evolution is only driven by GW emission. This assumption is of interest with the only aim of establishing an absolute upper 


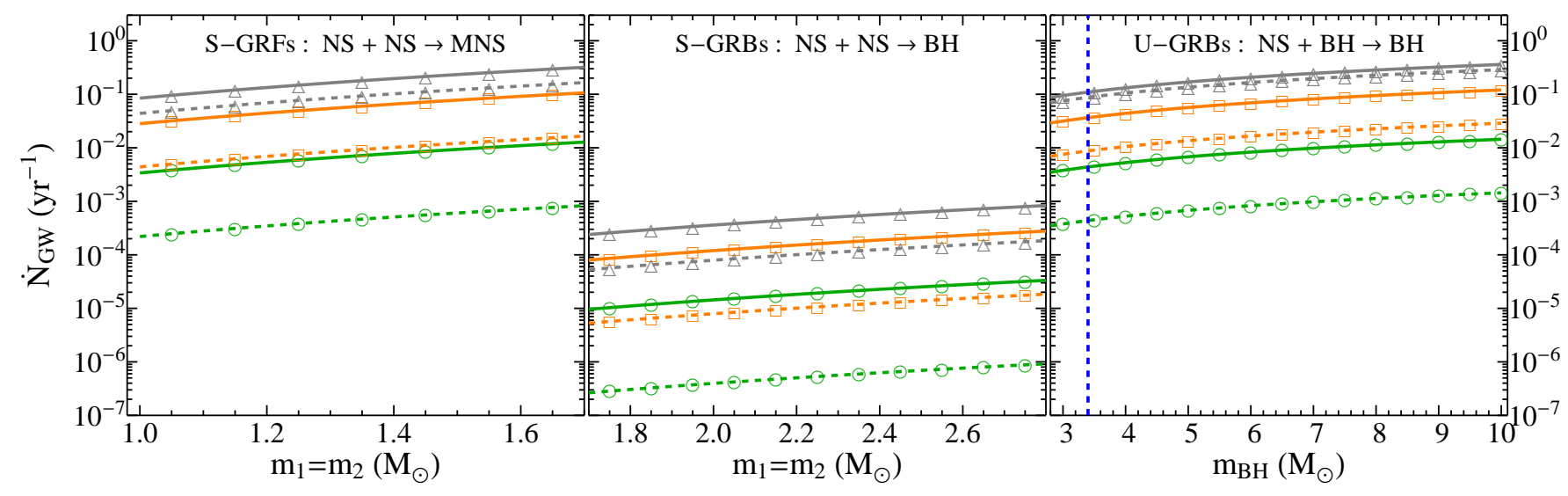

Figure 2. Expected annual GW upper and lower bounds (the solid and the dashed lines, respectively) for the detections expected from S-GRFs (left panel), S-GRBs (middle panel), and U-GRBs (right panel), for three selected observational campaigns: 2015/2016 (O1: red curves with circles), 2017/2018 (O3: orange curve with squares), and 2022+ (gray curve with triangles). The vertical red dashed line in the plot of U-GRBs separates $\nu$ NS-BH binaries produced by BdHN (BH masses equal to the NS critical mass) and BH-SN (BH masses larger than the NS critical mass).

Table 4. Column 1: GRB subclass. Column 2: inferred number of GRBs per year in the entire Universe, $\dot{N}_{\text {GRB }}$, for each GRB subclass (see also Fig. 6 in Ruffini et al. 2016b). Column 3: number of GRBs observed per year, $\dot{N}_{\mathrm{GRB}}^{\text {obs }}$, obtained from the observations of $\gamma$-ray telescopes such as AGILE, BATSE, BeppoSAX, Fermi, HETE-II, INTEGRAL, Konus/WIND and Swift, in the indicated years of observations (see also Tables 2-6 in Ruffini et al. 2016b). Column 4: expected rate of GW detections by Advanced LIGO of all the GRB subclasses, computed for three selected observational campaigns: 2015/2016 (O1), 2016/2017 (O2), 2017/2018 (O3) and the one by the entire network at design sensitivity including LIGO-India (2022+). The binary component masses used here are the same of Table 3.

\begin{tabular}{|c|c|c|c|}
\hline GRB subclass & $\dot{N}_{\mathrm{GRB}}\left(\mathrm{yr}^{-1}\right)$ & $\dot{N}_{\mathrm{GRB}}^{\mathrm{obs}}\left(\mathrm{yr}^{-1}\right)$ & $\dot{N}_{\mathrm{GW}}\left(\mathrm{yr}^{-1}\right)$ \\
\hline $\mathrm{BdHNe}$ & $662-1120$ & $14(1997-2014)$ & undetectable \\
\hline \multirow[t]{3}{*}{ S-GRFs } & $58-248$ & $3(2005-2014)$ & O1: $0.0001-0.002$ \\
\hline & & & O2: $0.002-0.01$ \\
\hline & & & $2022+: 0.1-0.2$ \\
\hline \multirow[t]{4}{*}{ S-GRBs } & $2-8$ & $1(2006-2014)$ & O1: $(0.1-3.1) \times 10^{-6}$ \\
\hline & & & O2: $(0.1-1.6) \times 10^{-5}$ \\
\hline & & & O3: $(0.6-7.8) \times 10^{-5}$ \\
\hline & & & $2022+:(0.78-3.12) \times 10^{-4}$ \\
\hline U-GRBs & & & $2022+: 0.076-0.094$ \\
\hline \multirow[t]{4}{*}{ U-GRBs (BH-SN) } & $\lesssim 662-1120$ & - & O1: $\lesssim 0.00036-0.0036$ \\
\hline & & & $\mathrm{O} 2: \lesssim 0.004-0.018$ \\
\hline & & & O3: $\lesssim 0.02-0.09$ \\
\hline & & & $2022+: \lesssim 0.29-0.36$ \\
\hline GRFs & $29-153$ & $1(2005-2014)$ & undetectable \\
\hline
\end{tabular}


limit and to check the detectability of the GW emission under this most optimistic condition. We compare and contrast in Sec. 5 the GW characteristic strain amplitude produced by the inspiral and merger regimes with the strain noise of the broadband detectors eLISA, Advanced LIGO-Virgo as well as of the narrow-band resonant bar NAUTILUS. In order to do this we use the cosmological redshift and corresponding luminosity distance of the closest observed source of each subclass (see Table 3). We show that the inspiral regime is possibly detectable only by Advanced LIGO (see Table 3 and Fig. 1) and the merger regime is undetectable by any of these instruments.

Therefore, in Sec. 6 we assess quantitatively the GW detectability of the inspiral regime of S-GRFs, S-GRBs and U-GRBs only by Advanced LIGO. We recall that, following Abbott et al. (2016), we adopt as the threshold for detectability a signal-to-noise ratio equal to 8 . We present in Fig. 2 and Table 4 the expected detection rate of the GW emission. Four observational campaigns of Advanced LIGO are analyzed: O1 (2015/2016), O2 (2016/2017), O3 (2017/2018), and 2022+ namely the one by the entire network at design sensitivity including LIGO-India. In Table 4 we compare and contrast this rate with the occurrence rate of the GRB subclasses and their rate of observations by $\gamma$-ray telescopes.

Keeping the above in mind, we conclude for each GRB subclass:

I. XRFs: their $\nu$ NS-NS out-states transit, during the inspiral regime which spans the frequency range $f<f_{\text {merger }} /(1+z)$ (see Table 3$)$, first the eLISA frequency band to then enter the Advanced LIGO-Virgo ones in the final orbits prior to the merging process (i.e. when $P_{\text {orb }}<0.2$ s). Resonant bar detectors are not sensitive in this inspiral regime frequency range. The characteristic strain generated by these sources in the inspiral regime is below the sensitivity of eLISA. The merger regime, which expands frequencies from $f_{\text {contact }} /(1+z)$ to $f_{\mathrm{qnm}} /(1+z)$ (see Table 3$)$, is outside the eLISA frequency band but inside the frequency band of Advanced LIGO-Virgo and bar detectors. See Fig. 1 for details. These $\nu$ NS-NS mergers can lead either to S-GRFs or S-GRBs (see in IV and V below the conclusion about their GW detectability).

II. BdHNe: their $\nu$ NS-BH out-states transit, during the inspiral regime which spans the frequency range $f<f_{\text {merger }} /(1+z)$ (see Table 3 ), first the eLISA frequency band to then enter the Advanced LIGO-Virgo ones in the final orbits prior to the merging process (i.e. when $P_{\text {orb }}<0.2$ s). Resonant bar detectors are not sensitive in this inspiral regime frequency range. The characteristic strain generated by these sources in the inspiral regime is below the sensitivity of eLISA. The merger regime, which expands frequencies from $f_{\text {contact }} /(1+z)$ to $f_{\mathrm{qnm}} /(1+z)$ (see Table 3 ), is outside the eLISA frequency band but inside the frequency band of Advanced LIGO-Virgo and bar detectors. See Fig. 1 for details. These $\nu$ NS-BH mergers lead to UGRBs (see in VI below the conclusion about their GW detectability).

III. BH-SN: their $\nu \mathrm{NS}-\mathrm{BH}$ out-states transit, during the inspiral regime which spans the frequency range $f<f_{\text {merger }} /(1+z)$ (see Table 3 ), first the eLISA frequency band to then enter the Advanced LIGO-Virgo ones in the final orbits prior to the merging process (i.e. when $P_{\text {orb }}<0.2$ s). Resonant bar detectors are not sensitive in this inspiral regime frequency range. The characteristic strain generated by these sources in the inspiral regime is below the sensitivity of eLISA. The merger regime, which expands frequencies from $f_{\text {contact }} /(1+z)$ to $f_{\mathrm{qnm}} /(1+z)$ (see Table 3 ), is outside the eLISA frequency band but inside the frequency band of Advanced LIGO-Virgo and bar detectors. See Fig. 1 for details. These $\nu \mathrm{NS}-\mathrm{BH}$ mergers lead to UGRBs (see in VI below the conclusion about their GW detectability).

IV. S-GRFs: the final orbits of the inspiral regime (when $P_{\text {orb }}<0.2 \mathrm{~s}$ ) fall inside the frequency band of Advanced LIGO-Virgo and bar detectors. However, the GW energy output in the merger regime leads to a characteristic strain which is not sufficient to be detectable either by any of them. See Fig. 1 for details. The inspiral regime is detectable for sources located at distances smaller than $181 \mathrm{Mpc}$ for the O1 Advanced LIGO run and smaller than $452 \mathrm{Mpc}$ for the $2022+$ run (see Table 3). The closest S-GRF observed up to now is, however, located at $509 \mathrm{Mpc}$. See Table 4 for the expected GW detection rate.

V. S-GRBs: the final orbits of the inspiral regime (when $P_{\text {orb }}<0.2 \mathrm{~s}$ ) fall inside the frequency band of Advanced LIGO-Virgo and bar detectors. However, the GW energy output in the merger regime leads to a characteristic strain which is not sufficient to be detectable either by any of them. See Fig. 1 for details. The inspiral regime is detectable for sources located at distances smaller than $244 \mathrm{Mpc}$ for the O1 Advanced LIGO run and smaller than $609 \mathrm{Mpc}$ for the 2022+ run (see Table 3). The closest S-GRB observed up to now is, however, located at $5842 \mathrm{Mpc}$. See Table 4 for the expected GW detection rate. 
VI. U-GRBs: the final orbits of the inspiral regime (when $P_{\text {orb }}<0.2 \mathrm{~s}$ ) fall inside the frequency band of Advanced LIGO-Virgo and bar detectors. However, the GW energy output in the merger regime leads to a characteristic strain which is not sufficient to be detectable either by any of them. See Fig. 1 for details. In the case of U-GRBs originating from the $\mathrm{BdHN}$ out-states, the inspiral regime is detectable for sources located at distances smaller than $253 \mathrm{Mpc}$ for the O1 Advanced LIGO run and smaller than $634 \mathrm{Mpc}$ for the $2022+$ run (see Table 3). In the case of U-GRBs originating from the $\mathrm{BH}-\mathrm{SN}$ out-states, the inspiral regime is detectable for sources at distances smaller than $396 \mathrm{Mpc}$ for the O1 Advanced LIGO run and smaller than $989 \mathrm{Mpc}$ for the $2022+$ run (see Table 3). No U-GRB has yet been electromagnetically identified. The closest distance at which is located its possible progenitor, namely a BdHN, is $805 \mathrm{Mpc}$. See Table 4 for the expected GW detection rate.

VII. GRFs: The tidal disruption of the WD by the NS produces a not detectable GW emission (see, e.g., Paschalidis et al. 2009).

We have recalled in the introduction that the simultaneous detection rate of GWs and GRBs have been estimated up to now in the literature only in the case of SGRFs, in which no BH is formed but instead the merger of the two NSs leads to a MNS. Indeed, it can be seen that the recent GW detection rate estimated by Patricelli et al. (2016) of short bursts at Advanced LIGO design sensitivity (see Table 1 there), $0.04-15 \mathrm{yr}^{-1}$, is consistent with the one of S-GRFs estimated in this work, $\dot{N}_{\mathrm{GW}}=0.1-0.2 \mathrm{yr}^{-1}$ (see Table 4). This represents the most favorable case for the possible GW detection by Advanced LIGO-Virgo of NS-NS merger which however does not lead to a $\mathrm{BH}$ formation but to a MNS.

We have given in this article, for the first time, a rate for the formation of BHs both in short and long bursts and this is of clear astrophysical relevance. Among such bursts producing a $\mathrm{BH}$, the most favorable cases for $\mathrm{GW}$ detection are those from U-GRBs from BdHNe with $\dot{N}_{\mathrm{GW}}=0.08-0.09 \mathrm{yr}^{-1}$ and those from BH-SN with $\dot{N}_{\mathrm{GW}}=0.3-0.4 \mathrm{yr}^{-1}$ (see Table 4 ). These NS-BH merging binaries were unknown in the literature and thus their occurrence and GW detection rates are a definite prediction of this work.

Any detection by Advanced LIGO-Virgo of a NS-NS merger or a NS-BH merger will imply a drastic increase of the occurrence rate of events shown here and an examination of the consistency with GRB observations.

We have already given evidence on the unsuitability of the collapsar model to explain the GRB observations in
Ruffini et al. (2018a). We have published a classification on the ground of the current observations of 480 sources with known redshift (Ruffini et al. 2018a, 2016b), which is both necessary and sufficient, as of today, to cover all GRBs observed. As the number of sources will increase it is conceivable that the discovery of different systems will be observed and in that case we will be ready for their inclusion in additional subclasses within our classification scheme.

As we have mentioned the above are estimates based on most favorable conditions for GW emission and realistic $\dot{N}_{\mathrm{GW}}$ values will need the assessment of the GW to electromagnetic energy ratio which is necessarily smaller than unity from energy conservation.

After the submission of this work, the LIGO-Virgo Collaboration announced the detection of the signal GW170817, and interpreted it as due to a NS-NS merger (Abbott et al. 2017b). As we have mentioned above, any possible GW detection of a NS-NS merger would imply a revision of its consistency with the inferences from GRB observations. It may then appear that our above conclusions of poor chance of detectability of NS-NS mergers by the Advanced LIGO-Virgo detector network are in tension with the detection of GW170817 during the O2 run. The association of GW170817 with GRB 170817A, a weak short-duration GRB observed by the Gamma-ray Burst Monitor (GBM) on board the Fermi-satellite (Abbott et al. 2017a; Goldstein et al. 2017), and followed-up in the optical bands (e.g. Cowperthwaite et al. 2017), in the X-rays (e.g. Haggard et al. 2017) as well as by further gamma-rays facilities (e.g. Savchenko et al. 2017), allows us in the following to make an assessment on this issue.

First, we recall that GRB $170817 \mathrm{~A}$, with its isotropic energy emitted in gamma-rays of $E_{\text {iso }} \approx 5 \times 10^{46} \mathrm{erg}$ (Goldstein et al. 2017) and peak luminosity of (1.7 \pm $0.1) \times 10^{47} \mathrm{erg} \mathrm{s}^{-1}$ (Zhang et al. 2017), would belong to the S-GRF subclass if we assume it is produced in a NS-NS merger. On the other hand, we recall that our estimates of the local density rate of the GRB subclasses (see Table 1), obtained from Ruffini et al. (2016b), are reliable for GRBs with luminosities higher than the lowest GRB luminosity in the subclass sample (see Appendix B for details). In the case of SGRFs, we had identified GRB 050509B as the source with the lowest energetics: $E_{\text {iso }} \approx 8.5 \times 10^{48} \mathrm{erg}$ (see Table 4 in Ruffini et al. 2016b) and a peak luminosity $(1.1 \pm 0.5) \times 10^{51} \mathrm{erg} \mathrm{s}^{-1}$ (Fox et al. 2005). This implies that our predicted detention rates for the Advanced LIGO-Virgo detectors for S-GRFs are valid for sources with electromagnetic emission over the above values. Even a single observation of a close and underluminous source, as GRB 170817A, would lead to an increase of the local density rate of this GRB subclass. 
Indeed, Zhang et al. (2017) have recently estimated the increase in the local density rate when GRB 170817A is included in the sample of short bursts. Following a similar method to the one described in the Appendix B, they found that their previously estimated isotropic local density rate of $(3.2-5.5) \mathrm{Gpc}^{-3} \mathrm{yr}^{-1}$, obtained for sources with peak luminosities above $7 \times 10^{49} \mathrm{erg} \mathrm{s}^{-1}, 3$ increases to a lower limit of (30-630) $\mathrm{Gpc}^{-3} \mathrm{yr}^{-1}$, for sources with peak luminosities above $1.7 \times 10^{47} \mathrm{erg} \mathrm{s}^{-1}$, i.e. when GW170817 is included in the sample. The above range implies an increase of the local density rate by a factor $\sim 10-100$. It is then easy to check, using our Table 4, that an increase of such a factor in the SGRF density rate would imply a detection rate of (0.011) $\mathrm{yr}^{-1}$ for the $\mathrm{O} 2$ observational run, in agreement with the detection of GW170817.

In fact, the above isotropic density rate inferred by Zhang et al. (2017) is consistent with the NS-NS observed merger rate of $(320-4740) \mathrm{Gpc}^{-3} \mathrm{yr}^{-1}$, in- ferred by the LIGO Collaboration with the detection of GW170817 (see Sec. V in Abbott et al. 2017b, for details). This result implies that S-GRFs (or in general all short bursts) are not beamed or, if a beaming is assumed, the jet half-opening angle should be at least as large as $25-30^{\circ}$.

\section{ACKNOWLEDGMENTS}

We thank the referee for suggestions which improved the presentation of our results. M.K. acknowledges the support given by the International Relativistic Astrophysics Erasmus Mundus Joint Doctorate Program under the Grants 2013-1471, from EACEA of the European Commission. M.M. and J.A.R. acknowledge the partial support of the project No 3101/GF4 IPC-11 and the target program F.0679 of the Ministry of Education and Science of the Republic of Kazakhstan. C.C. and S.F. acknowledge INdAM-GNFM for support.

\section{APPENDIX}

\section{A. IGC, HYPERCRITICAL ACCRETION, AND LONG GRBS}

We give in this appendix details of the accretion process within the IGC scenario following Fryer et al. (2014); Becerra et al. (2015); Fryer et al. (2015b); Becerra et al. (2016).

There are two main physical conditions for which hypercritical (i.e. highly super-Eddington) accretion onto the NS occurs in XRFs and BdHNe. The first is that the photons are trapped within the inflowing material and the second is that the shocked atmosphere on top the NS becomes sufficiently hot $\left(T \sim 10^{10} \mathrm{~K}\right)$ and dense $\left(\rho \gtrsim 10^{6} \mathrm{~g} \mathrm{~cm}^{-3}\right)$ to produce a very efficient neutrino-antineutrino $(\nu \bar{\nu})$ cooling emission. In this way the neutrinos become the main responsible to release the energy gained by accretion, allowing hypercritical accretion to continue.

The first IGC simulations were performed in Fryer et al. (2014), including: 1) realistic SN explosions of the $\mathrm{CO}_{\text {core }}$; 2) the hydrodynamics within the accretion region; 3) the simulated evolution of the SN ejecta up to their accretion onto the NS. Becerra et al. (2015) then estimated the amount of angular momentum carried by the SN ejecta and how much is transferred to the NS companion by accretion. They showed that the SN ejecta can circularize for a short time and form a disc-like structure surrounding the NS before being accreted. The evolution of the NS central density and rotation angular velocity (the NS is spun up by accretion) was computed from full numerical solutions of the axisymmetric Einstein equations. The unstable limits of the NS are set by the mass-shedding (or Keplerian) limit and the critical point of gravitational collapse given by the secular axisymmetric instability (see, e.g., Becerra et al. 2015, for details).

The accretion rate of the SN ejecta onto the NS is given by:

$$
\dot{M}_{B}(t)=\pi \rho_{\mathrm{ej}} R_{\mathrm{cap}}^{2} \sqrt{v_{\mathrm{rel}}^{2}+c_{\mathrm{s}, \mathrm{ej}}^{2}}, \quad R_{\mathrm{cap}}(t)=\frac{2 G M_{\mathrm{NS}}(t)}{v_{\mathrm{rel}}^{2}+c_{\mathrm{s}, \mathrm{ej}}^{2}},
$$

where $G$ is the gravitational constant, $\rho_{\mathrm{ej}}$ and $c_{\mathrm{s}, \mathrm{ej}}$ are the density and sound speed of the ejecta, $R_{\text {cap }}$ and $M_{\mathrm{NS}}$ are the NS gravitational capture radius (Bondi-Hoyle radius) and gravitational mass, and $v_{\text {rel }}$ the ejecta velocity relative to the NS: $\vec{v}_{\text {rel }}=\vec{v}_{\text {orb }}-\vec{v}_{\text {ej }} ;\left|\vec{v}_{\text {orb }}\right|=\sqrt{G\left(M_{\text {core }}+M_{\mathrm{NS}}\right) / a}$, and $\vec{v}_{\text {ej }}$ is the velocity of the supernova ejecta (see Fig. A1).

Numerical simulations of the SN explosions suggest the adopted homologous expansion of the SN, i.e. $v_{\mathrm{ej}}(r, t)=n r / t$, where $r$ is the position of each layer from the SN center and $n$ is the expansion parameter. The density evolves as

$$
\rho_{\mathrm{ej}}(r, t)=\rho_{\mathrm{ej}}^{0}\left(r / R_{\mathrm{star}}(t), t_{0}\right) \frac{M_{\mathrm{env}}(t)}{M_{\mathrm{env}}(0)}\left(\frac{R_{\mathrm{star}}(0)}{R_{\mathrm{star}}(t)}\right)^{3},
$$

\footnotetext{
3 This rate is consistent with the local density rate $\rho_{\mathrm{S}-\mathrm{GRFs}}+$ $\rho_{\mathrm{S}-\mathrm{GRBs}} \approx \rho_{\mathrm{S}-\mathrm{GRFs}}=(2.6-5.0) \mathrm{Gpc}^{-3} \mathrm{yr}^{-1}$ used in the present
} 


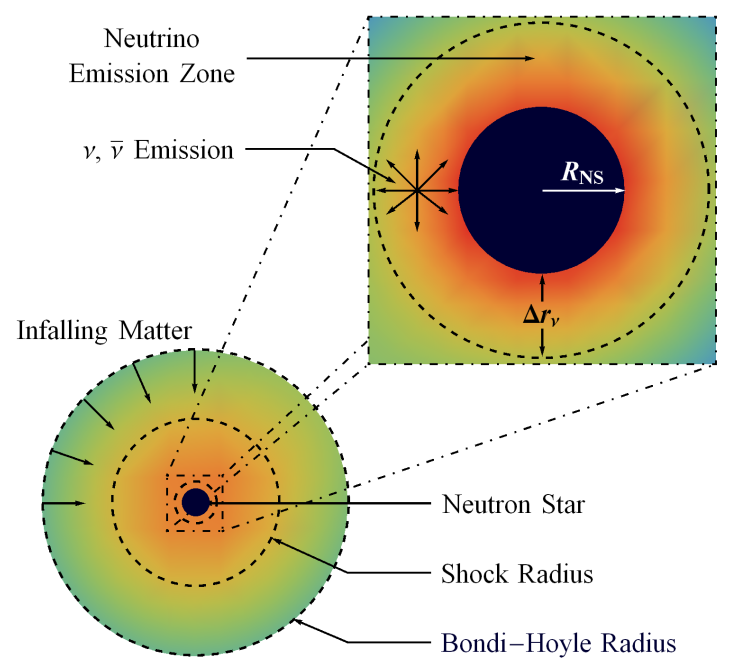

Figure A1. Scheme of the IGC scenario: the $\mathrm{CO}_{\text {core }}$ undergoes SN explosion, the NS accretes part of the SN ejecta and then reaches the critical mass for gravitational collapse to a $\mathrm{BH}$, with consequent emission of a GRB. The SN ejecta reach the NS Bondi-Hoyle radius and fall toward the NS surface. The material shocks and decelerates while it piles over the NS surface. At the neutrino emission zone, neutrinos take away most of the gravitational energy gained by the matter infall. The neutrinos are emitted above the NS surface in a region of thickness $\Delta r_{\nu}$ about half the NS radius that allow the material to reduce its entropy to be finally incorporated to the NS. For further details and numerical simulations of the above process see Fryer et al. (2014); Becerra et al. (2015, 2016).

where $M_{\text {env }}(t)$ the mass of the $\mathrm{CO}_{\text {core }}$ envelope, $R_{\mathrm{star}}(t)$ is the radius of the outermost layer, and $\rho_{\mathrm{ej}}^{0}$ is the pre-SN $\mathrm{CO}_{\text {core }}$ density profile; $\rho_{\mathrm{ej}}\left(r, t_{0}\right)=\rho_{\text {core }}\left(R_{\text {core }} / r\right)^{m}$, where $\rho_{\text {core }}, R_{\text {core }}$ and $m$ are the profile parameters obtained from numerical simulations. Typical parameters of the $\mathrm{CO}_{\text {core }}$ mass are (3.5-9.5) $M_{\odot}$ corresponding to (15-30) $M_{\odot}$ zeroage-main-sequence (ZAMS) progenitors (see Fryer et al. 2014; Becerra et al. 2015, for details). The binary period is limited from below by the request of having no Roche lobe overflow by the $\mathrm{CO}_{\text {core }}$ before the $\mathrm{SN}$ explosion (Fryer et al. 2014). For instance, for a $\mathrm{CO}_{\text {core }}$ of $9.5 M_{\odot}$ forming a binary system with a $2 M_{\odot} \mathrm{NS}$, the minimum orbital period allowed by this condition is $P_{\min } \approx 5 \mathrm{~min}$. For these typical binary and pre-SN parameters, Eq. (A1) gives accretion rates $10^{-4}-10^{-2} M_{\odot} \mathrm{s}^{-1}$.

We adopt an initially non-rotating NS companion so its exterior spacetime at time $t=0$ is described by the Schwarzschild metric. The SN ejecta approach the NS with specific angular momentum, $l_{\text {acc }}=\dot{L}_{\text {cap }} / \dot{M}_{B}$, circularizing at a radius $r_{\text {circ }} \geq r_{\text {lco }}$ if $l_{\text {acc }} \geq l_{\text {lso }}$ with $r_{\text {lco }}$ the radius of the last circular orbit (LCO). For a non-rotating NS $r_{\text {lco }}=6 G M_{\mathrm{NS}} / c^{2}$ and $l_{\text {lco }}=2 \sqrt{3} G M_{\mathrm{NS}} / c$. For typical parameters, $r_{\text {circ }} / r_{\text {lco }} \sim 10-10^{3}$.

The accretion onto the NS proceeds from the radius $r_{\text {in }}$. The Ns mass and angular angular momentum evolve as (Becerra et al. 2015; Cipolletta et al. 2017):

$$
\dot{M}_{\mathrm{NS}}=\left(\frac{\partial M_{\mathrm{NS}}}{\partial M_{b}}\right)_{J_{\mathrm{NS}}} \dot{M}_{b}+\left(\frac{\partial M_{\mathrm{NS}}}{\partial J_{\mathrm{NS}}}\right)_{M_{b}} \dot{J}_{\mathrm{NS}}, \quad \dot{J}_{\mathrm{NS}}=\xi l\left(r_{\mathrm{in}}\right) \dot{M}_{\mathrm{B}}
$$

where $M_{b}$ is the NS baryonic mass, $l\left(r_{\text {in }}\right)$ is the specific angular momentum of the accreted material at $r_{\text {in }}$, which corresponds to the angular momentum of the LCO, and $\xi \leq 1$ is a parameter that measures the efficiency of angular momentum transfer. In this picture we have $\dot{M}_{b}=\dot{M}_{B}$.

For the integration of Eqs. (A1) and (A3) we have to supply the values of the two partial derivatives in Eq. (A3). They are obtained from the relation of the NS gravitational mass, $M_{\mathrm{NS}}$, with $M_{b}$ and $J_{\mathrm{NS}}$, namely from the knowledge of the NS binding energy. For this we use the general relativistic calculations of rotating NSs presented in Cipolletta et al. (2015). They show that, independent on the nuclear EOS, the following analytical formula represents the numerical results with sufficient accuracy (error $<2 \%$ ):

$$
\frac{M_{b}}{M_{\odot}}=\frac{M_{\mathrm{NS}}}{M_{\odot}}+\frac{13}{200}\left(\frac{M_{\mathrm{NS}}}{M_{\odot}}\right)^{2}\left(1-\frac{1}{137} j_{\mathrm{NS}}^{1.7}\right)
$$

where $j_{\mathrm{NS}} \equiv c J_{\mathrm{NS}} /\left(G M_{\odot}^{2}\right)$.

In the accretion process the NS gains angular momentum and therefore spin up. To evaluate the amount of angular 
Table A1. Critical NS mass in the non-rotating case and constants $k$ and $p$ needed to compute the NS critical mass in the non-rotating case given by Eq. (A6). The values are given for the NL3, GM1 and TM1 EOS.

\begin{tabular}{cccc}
\hline \hline EOS & $M_{\text {crit }}^{J=0}\left(M_{\odot}\right)$ & $p$ & $k$ \\
\hline NL3 & 2.81 & 1.68 & 0.006 \\
GM1 & 2.39 & 1.69 & 0.011 \\
TM1 & 2.20 & 1.61 & 0.017 \\
\hline \hline
\end{tabular}

momentum transferred to the NS at any time we include the dependence of the LCO specific angular momentum as a function of $M_{\mathrm{NS}}$ and $J_{\mathrm{NS}}$. For corotating orbits the following relation is valid for the NL3, TM1 and GM1 EOS (Cipolletta et al. 2017; Becerra et al. 2015):

$$
l_{\mathrm{lco}}=\frac{G M_{\mathrm{NS}}}{c}\left[2 \sqrt{3}-0.37\left(\frac{j_{\mathrm{NS}}}{M_{\mathrm{NS}} / M_{\odot}}\right)^{0.85}\right] .
$$

The NS continues to accrete until an instability limit is reached or up to when all the SN ejecta overcomes the NS Bondi-Hoyle region. We take into account the two main instability limits for rotating NSs: the mass-shedding or Keplerian limit and the secular axisymmetric instability limit. The latter defines critical NS mass. For the aforementioned nuclear EOS, the critical mass is approximately given by (Cipolletta et al. 2015):

$$
M_{\mathrm{NS}}^{\mathrm{crit}}=M_{\mathrm{NS}}^{J=0}\left(1+k j_{\mathrm{NS}}^{p}\right),
$$

where $k$ and $p$ are EOS-dependent parameters (see Table A1). These formulas fit the numerical results with a maximum error of $0.45 \%$.

\section{A.1. Most recent simulations of the IGC process}

Additional details and improvements of the hypercritical accretion process leading to XRFs and BdHNe were presented in Becerra et al. (2016). Specifically:

1. The density profile included finite size/thickness effects and additional $\mathrm{CO}_{\text {core }}$ progenitors leading to different SN ejecta masses were considered.

2. In Becerra et al. (2015) the maximum orbital period, $P_{\max }$, over which the accretion onto NS companion is not sufficient to bring it to the critical mass, was inferred. Thus, binaries with $P>P_{\max }$ lead to XRFs while the ones with $P \lesssim P_{\max }$ lead to BdHNe. Becerra et al. (2016) extended the determination of $P_{\max }$ for all the possible initial values of the NS mass. They also examined the outcomes for different values of the angular momentum transfer efficiency parameter.

3. It was estimated the expected luminosity during the process of hypercritical accretion for a wide range of binary periods covering both XRFs and BdHNe.

4. It was shown that the presence of the NS companion originates asymmetries in the SN ejecta (see, e.g., Fig. 6 in Becerra et al. 2016). The signatures of such asymmetries in the X-ray emission was there shown in the specific example of XRF 060218.

\section{A.2. Hydrodynamics and neutrino emission in the accretion region}

The accretion rate onto the NS can be as high as $\sim 10^{-2}-10^{-1} M_{\odot} \mathrm{s}^{-1}$. For such accretion rates:

1. The magnetic pressure is much smaller than the random pressure of the infalling material, therefore the magneticfield effects on the accretion process are negligible (Fryer et al. 1996; Rueda \& Ruffini 2012).

2. The photons are trapped within the infalling matter, hence the Eddington limit does not apply and hypercritical accretion occurs. The trapping radius is defined by (Chevalier 1989): $r_{\text {trapping }}=\min \left\{\dot{M}_{B} \kappa /(4 \pi c), R_{\text {cap }}\right\}$, where $\kappa$ is the opacity. Fryer et al. (2014) estimated a Rosseland mean opacity of $\approx 5 \times 10^{3} \mathrm{~cm}^{2} \mathrm{~g}^{-1}$ for the $\mathrm{CO}_{\text {cores }}$. This, together with our typical accretion rates, lead to $\dot{M}_{B} \kappa /(4 \pi c) \sim 10^{13}-10^{19} \mathrm{~cm}$. This radius is much bigger than the Bondi-Hoyle radius.

3. The above condition, and the temperature-density values reached on top the NS surface, leads to an efficient neutrino cooling which radiates away the gain of gravitational energy of the infalling material (Zel'dovich et al. 1972; Ruffini \& Wilson 1973; Fryer et al. 1996; Rueda \& Ruffini 2012; Fryer et al. 2014). 


\section{A.2.1. Convective instabilities}

The accretion shock moves outward as the material piles onto the NS. Since the post-shock entropy is inversely proportional to the shock radius position the NS atmosphere is unstable with respect to Rayleigh-Taylor convection at the beginning of the accretion process. Such instabilities might drive high-velocity outflows from the accreting NS (Fryer et al. 2006; Fryer 2009). The entropy at the base of the atmosphere is (Fryer et al. 1996):

$$
S_{\text {bubble }} \approx 16\left(\frac{1.4 M_{\odot}}{M_{\mathrm{NS}}}\right)^{-7 / 8}\left(\frac{M_{\odot} \mathrm{s}^{-1}}{\dot{M}_{\mathrm{B}}}\right)^{1 / 4}\left(\frac{10^{6} \mathrm{~cm}}{r}\right)^{3 / 8} k_{B} / \text { nucleon },
$$

The material expands and cools down adiabatically, i.e. $T^{3} / \rho=$ constant. In the case of a spherically symmetric expansion, $\rho \propto 1 / r^{3}$ and $k_{B} T_{\text {bubble }}=195 S_{\text {bubble }}^{-1}\left(10^{6} \mathrm{~cm} / r\right) \mathrm{MeV}$. In the more likely case that the material expand laterally we have (Fryer 2009): $\rho \propto 1 / r^{2}$, i.e. $T_{\text {bubble }}=T_{0}\left(S_{\text {bubble }}\right)\left(r_{0} / r\right)^{2 / 3}$, where $T_{0}\left(S_{\text {bubble }}\right)$ is obtained from the above equation at $r=r_{0} \approx R_{\mathrm{NS}}$. This implies a bolometric blackbody flux at the source from the rising bubbles:

$$
F_{\text {bubble }} \approx 2 \times 10^{40}\left(\frac{M_{\mathrm{NS}}}{1.4 M_{\odot}}\right)^{-7 / 2}\left(\frac{\dot{M}_{\mathrm{B}}}{M_{\odot} \mathrm{s}^{-1}}\right)\left(\frac{R_{\mathrm{NS}}}{10^{6} \mathrm{~cm}}\right)^{3 / 2}\left(\frac{r_{0}}{r}\right)^{8 / 3} \mathrm{erg} \mathrm{s}^{-1} \mathrm{~cm}^{-2},
$$

where $\sigma$ is the Stefan-Boltzmann constant.

The above thermal emission has been shown (Fryer et al. 2014) to be a plausible explanation of the early $(t \lesssim 50 \mathrm{~s})$ X-ray emission observed in some GRBs. In the specific example of GRB 090618 (Izzo et al. 2012b,a), adopting an accretion rate of $10^{-2} M_{\odot} \mathrm{s}^{-1}$, the bubble temperature drops from $50 \mathrm{keV}$ to $15 \mathrm{keV}$ while expanding from $r \approx 10^{9} \mathrm{~cm}$ to $6 \times 10^{9} \mathrm{~cm}$.

\section{A.2.2. Neutrino emission and effective accretion rate}

Temperatures $k_{B} T \sim 1-10 \mathrm{MeV}$ and densities $\rho \gtrsim 10^{6} \mathrm{~g} \mathrm{~cm}^{-3}$ develop near the NS surface during the accretion process. Under these conditions, $e^{+} e^{-}$annihilation into $\nu \bar{\nu}$ pairs becomes the dominant neutrino emission process in the accretion region (see Becerra et al. 2016, for details). The effective accretion rate onto the NS can be estimated as (Fryer et al. 1996, e.g.): $\dot{M}_{\text {eff }} \approx \Delta M_{\nu}\left(L_{\nu} / E_{\nu}\right)$, where $\Delta M_{\nu}$ and $L_{\nu}$ are the mass and neutrino luminosity in the emission region; $E_{\nu}$ is half the gravitational potential energy gained by the material falling from infinity to a distance $\Delta r_{\nu}$ from the NS surface. $\Delta r_{\nu}$ is the thickness of the neutrino emitting region which is approximately given by the temperature scale height $\left(\Delta r_{\nu} \approx 0.6 R_{\mathrm{NS}}\right)$. Since $L_{\nu} \approx 2 \pi R_{\mathrm{NS}}^{2} \Delta r_{\nu} \epsilon_{\mathrm{e}^{-} \mathrm{e}^{+}}$with $\epsilon_{\mathrm{e}^{-} \mathrm{e}^{+}}$the $e^{+} e^{-}$pair annihilation process emissivity, and $E_{\nu}=(1 / 2) G M_{\mathrm{NS}} \Delta M_{\nu} /\left(R_{\mathrm{NS}}+\Delta r_{\nu}\right)$, for $M_{\mathrm{NS}}=1.4 M_{\odot}$ one obtains $\dot{M}_{\mathrm{eff}} \approx 10^{-9}-10^{-1} M_{\odot} \mathrm{s}^{-1}$ for $k_{B} T=1-10 \mathrm{MeV}$.

\section{A.3. Accretion luminosity}

The energy release in a time-interval $d t$, when an amount of mass $d M_{b}$ with angular momentum $l \dot{M}_{b}$ is accreted, is:

$$
L_{\mathrm{acc}}=\left(\dot{M}_{b}-\dot{M}_{\mathrm{NS}}\right) c^{2}=\dot{M}_{b} c^{2}\left[1-\left(\frac{\partial M_{\mathrm{NS}}}{\partial J_{\mathrm{NS}}}\right)_{M_{b}} l-\left(\frac{\partial M_{\mathrm{NS}}}{\partial M_{b}}\right)_{J_{\mathrm{NS}}}\right] .
$$

This is the amount of gravitational energy gained by the matter by infalling to the NS surface that is not spent in NS gravitational binding energy. The total energy release in the time interval from $t$ to $t+d t, \Delta E_{\text {acc }} \equiv \int L_{\text {acc }} d t$, is given by the NS binding energy difference between its initial and final state. The typical luminosity is $L_{\text {acc }} \approx \Delta E_{\text {acc }} / \Delta t_{\text {acc }}$, where $\Delta t_{\text {acc }}$ is the duration of the accretion process.

The value of $\Delta t_{\text {acc }}$ is approximately given by the flow time of the slowest layers of the SN ejecta to the NS companion

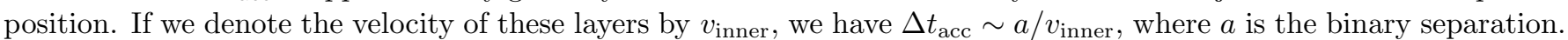
For $a \sim 10^{11} \mathrm{~cm}$ and $v_{\text {inner }} \sim 10^{8} \mathrm{~cm} \mathrm{~s}^{-1}, \Delta t_{\text {acc }} \sim 10^{3}$ s. For shorter separations, e.g. $a \sim 10^{10} \mathrm{~cm}(P \sim 5 \mathrm{~min})$, $\Delta t_{\text {acc }} \sim 10^{2} \mathrm{~s}$. For a binary with $P=5 \mathrm{~min}$, the NS accretes $\approx 1 M_{\odot}$ in $\Delta t_{\text {acc }} \approx 100 \mathrm{~s}$. From Eq. (A4) one obtains that the binding energy difference of a $2 M_{\odot}$ and a $3 M_{\odot} \mathrm{NS}$, is $\Delta E_{\text {acc }} \approx 13 / 200\left(3^{2}-2^{2}\right) M_{\odot} c^{2} \approx 0.32 M_{\odot} c^{2}$. This leads to $L_{\text {acc }} \approx 3 \times 10^{-} 3 M_{\odot} c^{2} \approx 0.1 \dot{M}_{b} c^{2}$. The accretion power can be as high as $L_{\text {acc }} \sim 0.1 \dot{M}_{b} c^{2} \sim 10^{47}-10^{51}$ erg s ${ }^{-1}$ for accretion rates in the range $\dot{M}_{b} \sim 10^{-6}-10^{-2} M_{\odot} \mathrm{s}^{-1}$.

\section{A.4. Possible evolutionary scenario for $C O_{\text {core }}-N S$ binary formation}

Two independent communities have introduced a new evolutionary scenario for the formation of compact-object binaries (NS-NS or NS-BH). After the collapse of the primary star forming a NS, the binary undergoes mass-transfer episodes finally leading to the ejection of both the hydrogen and helium shells of the secondary star. These processes 
leads naturally to a binary composed of a $\mathrm{CO}_{\text {core }}$ and a $\mathrm{NS}$ companion. In the X-ray binary and $\mathrm{SN}$ communities these systems are called "ultra-stripped" binaries (Tauris et al. 2015a, see, e.g.,). These systems are expected to comprise 0.1-1\% of the total SNe (Tauris et al. 2013).

In the above studies most of the binaries have orbital periods in the range $3 \times 10^{3}-3 \times 10^{5} \mathrm{~s}$ which are longer with

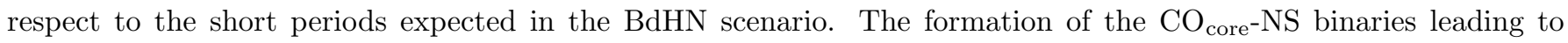
BdHNe might be a subset of the ultra-stripped binaries. In such subset the conditions of the initial orbital separation and $\mathrm{CO}_{\text {core }}$ mass must be such to lead to final orbital periods in the range 100-1000 s. Assuming a SN rate of $2 \times 10^{4} \mathrm{Gpc}^{-3} \mathrm{yr}^{-1}$ (Guetta \& Della Valle 2007), the ultra-stripped binaries would have a rate of 20-200 $\mathrm{Gpc}^{-3} \mathrm{yr}^{-1}$, and thus BdHNe, with a rate of $\sim 1 \mathrm{Gpc}^{-3} \mathrm{yr}^{-1}$ (see Table 1 and Ruffini et al. 2016b), might be produced by the $0.5-5 \%$ of the ultra-stripped binary population.

\section{A.5. Post-Explosion Orbits and NS-BH Binaries formation}

The SN explosion leaves as a central remnant the $\nu \mathrm{NS}$, while the NS companion might lead, for sufficient accretion rates, to the formation of a BH. We examined in Fryer et al. (2015b) the question if BdHNe can indeed form NS-BH binaries or, on the contrary, they are disrupted by the SN explosion.

Most of the typical binaries become unbound during a SN explosion owing to the amount of mass loss and momentum imparted (kick) to the $\nu$ NS in the explosion. Assuming an instantaneous explosion, the binary is disrupted if half of the binary mass is lost. For this reason the fraction of massive binaries that can produce double compact-object binaries might be as low as 0.001-1\% (Fryer et al. 1999a; Dominik et al. 2012; Postnov \& Yungelson 2014). Indeed, this is consistent with our estimated GRB local observed rates: we have shown in section 3.1 that the NS-NS population leading to short-bursts can be explained as being descendant from the $\mathrm{CO}_{\text {core }} \mathrm{NS}$ if $\sim 1 \%$ of them remain bound after the SN explosion.

Assuming instantaneous mass loss, the post-explosion semi-major axis is (Hills 1983):

$$
\frac{a}{a_{0}}=\frac{M_{0}-\Delta M}{M_{0}-2 a_{0} \Delta M / r},
$$

where $a_{0}$ and $a$ are the initial and final semi-major axes respectively, $M_{0}$ is the (initial) binary mass, $\Delta M$ is the change of mass (in this case the amount of mass loss), and $r$ is the orbital separation before the explosion. For circular orbits, the system is unbound if it loses half of its mass. For the very tight BdHNe, however, additional effects have to be taken into account to determine the fate of the binary.

The shock front in a SN moves at roughly $10^{4} \mathrm{~km} \mathrm{~s}^{-1}$, but the denser, lower-velocity ejecta, can move at velocities as low as $10^{2}-10^{3} \mathrm{~km} \mathrm{~s}^{-1}$ (Fryer et al. 2014). This implies that the SN ejecta overcomes a NS companion in a time 10-1000 s. For wide binaries this time is a small fraction of the orbital period and the "instantaneous" mass-loss assumption is perfectly valid. BdHNe have instead orbital periods as short as 100-1000 s, hence the instantaneous mass-loss approximation breaks down.

We recall the specific examples studied in Fryer et al. (2015b): close binaries in an initial circular orbit of radius $7 \times 10^{9} \mathrm{~cm}, \mathrm{CO}_{\text {core }}$ radii of $(1-4) \times 10^{9} \mathrm{~cm}$ with a $2.0 M_{\odot} \mathrm{NS}$ companion. The $\mathrm{CO}_{\text {core }}$ leaves a central $1.5 M_{\odot} \mathrm{NS}$, ejecting the rest of the core. The NS leads to a $\mathrm{BH}$ with a mass equal to the NS critical mass. For these parameters it was there obtained that even if $70 \%$ of the mass is lost the binary remains bound providing the explosion time is of the order of the orbital period $(P=180 \mathrm{~s})$ with semi-major axes of less than $10^{11} \mathrm{~cm}$.

The tight $\nu \mathrm{NS}-\mathrm{BH}$ binaries produced by BdHNe will, in due time, merge owing to the emission of GWs. For the above typical parameters the merger time is of the order of $10^{4} \mathrm{yr}$, or even less. We expect little baryonic contamination around such merger site since this region has been cleaned-up by the BdHN. These conditions lead to a new family of sources which we have called ultrashort GRBs, U-GRBs.

\section{B. LOCAL DENSITY RATE OF GRB SUBCLASSES}

We recall now the method used in Ruffini et al. (2016b) to estimate, for each GRB subclass, the local observed density rates that we use in this work. This is defined by the convolution of the luminosity function, that tells us the fraction of bursts with isotropic equivalent luminosities in the interval $\log L$ and $\log L+d \log L$, and the cosmic GRB occurrence rate, that tells us the number of sources at different redshifts. These functions depend on a priori assumptions and some investigations have been carried out in the literature: for long bursts (e.g. Soderberg et al. 2006a; Guetta \& Della Valle 2007; Liang et al. 2007; Virgili et al. 2009; Rangel Lemos et al. 2010; Wanderman \& Piran 2010; Guetta et al. 2011; Kovacevic et al. 2014)), for short bursts (e.g. Virgili et al. 2011; Wanderman \& Piran 2015), and for both long and short bursts (e.g. Sun et al. 2015). Additional properties that introduce further uncertainties 
are the instrumental sensitivity threshold, the field of view $\Omega_{i}$, and the operational time $T_{i}$ of $i$-detector.

Hereafter we neglect the possible redshift-evolution of the luminosity function. For $\Delta N_{i}$ events detected by various detectors in a finite $\operatorname{logarithmic} \operatorname{luminosity}$ bin from $\log L$ to $\log L+\Delta \log L$, the total local event rate density between observed minimum, $L_{\min }$, and maximum, $L_{\max }$, luminosities, is (e.g. Sun et al. 2015)

$$
\rho_{0} \simeq \sum_{i} \sum_{\log L_{\min }}^{\log L_{\max }} \frac{4 \pi}{\Omega_{i} T_{i}} \frac{1}{\ln 10} \frac{1}{g(L)} \frac{\Delta N_{i}}{\Delta \log L} \frac{\Delta L}{L},
$$

where

$$
g(L)=\int_{0}^{z_{\max }(L)} \frac{f(z)}{1+z} \frac{d V(z)}{d z} d z
$$

and the comoving volume is

$$
\frac{d V(z)}{d z}=\frac{c}{H_{0}} \frac{4 \pi d_{L}^{2}}{(1+z)^{2}\left[\Omega_{M}(1+z)^{3}+\Omega_{\Lambda}\right]^{1 / 2}},
$$

where $d_{L}$ is the luminosity distance. We set $f(z)=1$, namely we do not assume any redshift dependence of the GRB cosmic event rate density. The maximum volume within which the event of luminosity $L$ can be detected is defined by the maximum redshift $z_{\max }(L)$. The latter is computed, following Schaefer (2007), from the $1 \mathrm{~s}$-bolometric peak luminosity $L, k$-corrected from the observed detector energy band into the burst cosmological rest-frame energy band 1-10 $\mathrm{keV}$, and the corresponding $1 \mathrm{~s}$-threshold peak flux $f_{\mathrm{th}}$. This is the limiting peak flux for the burst detection (Band 2003). With this, $z_{\max }$ can be defined from (see, e.g., Zhang et al. 2009; Ruffini et al. 2014)

$$
f_{\mathrm{th}}=\frac{L}{4 \pi d_{L}^{2}\left(z_{\max }\right) k} \text {. }
$$

The possible evolution with the redshift of the GRB density rates have been analyzed in Ruffini et al. (2016b) by separating the bursts into several redshift bins, following the method suggested in Sun et al. (2015). In each redshift interval $z_{j} \leq z \leq z_{j+1}$, the integration limits of Eq. (B12) are replaced by $z_{j}$ and $\min \left[z_{j+1}, z_{\max , \mathrm{j}}(L)\right]$, where $z_{\max , \mathrm{j}}(L)$ is the maximum redshift for the $\mathrm{j}^{\text {th }}$ redshift bin. Finally, from Eq. (B11) an event rate $\rho_{0}^{\mathrm{z}}$ in each redshift bin around $z$ is obtained.

We adopt the fields of view and operational times for the detectors: Beppo-SAX, $\Omega_{\mathrm{BS}}=0.25 \mathrm{sr}, T_{\mathrm{BS}}=7 \mathrm{y}$; BATSE, $\Omega_{\mathrm{B}}=\pi \mathrm{sr}, T_{\mathrm{B}}=10 \mathrm{y}$, HETE- $2, \Omega_{\mathrm{H}}=0.8 \mathrm{sr}, T_{\mathrm{H}}=7 \mathrm{y} ;$ Swift-BAT, $\Omega_{\mathrm{S}}=1.33 \mathrm{sr}, T_{\mathrm{S}}=10 \mathrm{y} ;$ Fermi-GBM, $\Omega_{\mathrm{F}}=9.6 \mathrm{sr}$, $T_{\mathrm{F}}=7 \mathrm{y}$. We adopt no beaming correction.

\section{REFERENCES}

Abbott, B. P., Abbott, R., Abbott, T. D., et al. 2016, Living Reviews in Relativity, 19, arXiv:1304.0670

—. 2017a, ApJL, 848, L13

—. 2017b, Physical Review Letters, 119, 161101

Aimuratov, Y., Ruffini, R., Muccino, M., et al. 2017a, ApJ, 844, 83

—. 2017b, ArXiv: 1704.08179, arXiv:1704.08179

Allen, B., Anderson, W. G., Brady, P. R., Brown, D. A., \& Creighton, J. D. E. 2012, PhRvD, 85, 122006

Anninos, P., Hobill, D., Seidel, E., Smarr, L., \& Suen, W.-M. 1995, PhRvD, 52, 2044

Antoniadis, J., Freire, P. C. C., Wex, N., et al. 2013, Science, 340, 448

Astone, P., Ballantini, R., Babusci, D., et al. 2006, Classical and Quantum Gravity, 23, S57

-. 2008, Classical and Quantum Gravity, 25, 114048

Band, D. L. 2003, ApJ, 588, 945

Becerra, L., Bianco, C. L., Fryer, C. L., Rueda, J. A., \& Ruffini, R. 2016, ApJ, 833, 107

Becerra, L., Cipolletta, F., Fryer, C. L., Rueda, J. A., \& Ruffini, R. 2015, ApJ, 812, 100

Belczynski, K., Bulik, T., \& Bailyn, C. 2011, ApJL, 742, L2

Belczynski, K., Bulik, T., \& Rudak, B. 2002, ApJ, 571, 394
Belczynski, K., Repetto, S., Holz, D. E., et al. 2016, ApJ, 819, 108

Belvedere, R., Boshkayev, K., Rueda, J. A., \& Ruffini, R. 2014, Nucl. Phys. A, 921, 33

Berger, E. 2014, ARA\&A, 52, 43

Bernuzzi, S., Dietrich, T., \& Nagar, A. 2015, Phys. Rev. Lett., 115, 091101

Bildsten, L., \& Cutler, C. 1992, ApJ, 400, 175

Cadelano, M., Pallanca, C., Ferraro, F. R., et al. 2015, ApJ, 812, 63

Caito, L., Bernardini, M. G., Bianco, C. L., et al. 2009, A\&A, 498, 501

Camp, J. B., \& Cornish, N. J. 2004, Annual Review of Nuclear and Particle Science, 54, 525

Chevalier, R. A. 1989, ApJ, 346, 847

Cipolletta, F., Cherubini, C., Filippi, S., Rueda, J. A., \& Ruffini, R. 2015, PhRvD, 92, 023007

—. 2017, PhRvD, 96, 024046

Clark, J. P. A., \& Eardley, D. M. 1977, ApJ, 215, 311

Cowperthwaite, P. S., Berger, E., Villar, V. A., et al. 2017, ApJL, 848, L17

Dai, Z. G., \& Lu, T. 1998a, A\&A, 333, L87

—. 1998b, Physical Review Letters, 81, 4301 
Davis, M., Ruffini, R., Press, W. H., \& Price, R. H. 1971, Phys. Rev. Lett., 27, 1466

Davis, M., Ruffini, R., \& Tiomno, J. 1972, PhRvD, 5, 2932

Della Valle, M., Chincarini, G., Panagia, N., et al. 2006, Nature, 444, 1050

Detweiler, S. 1980, ApJ, 239, 292

Detweiler, S. L., \& Szedenits, Jr., E. 1979, ApJ, 231, 211

Dominik, M., Belczynski, K., Fryer, C., et al. 2012, ApJ, 759, 52

Dominik, M., Berti, E., O'Shaughnessy, R., et al. 2015, ApJ, 806, 263

Dutra, M., Lourenço, O., \& Menezes, D. P. 2016, Phys. Rev. C, 93, 025806

Dutra, M., Lourenço, O., Avancini, S. S., et al. 2014, Phys. Rev. C, 90, 055203

Eichler, D., Livio, M., Piran, T., \& Schramm, D. N. 1989, Nature, 340, 126

Finn, L. S., \& Chernoff, D. F. 1993, PhRvD, 47, 2198

Flanagan, É. É., \& Hughes, S. A. 1998, PhRvD, 57, 4535

Fong, W., Berger, E., Margutti, R., \& Zauderer, B. A. 2015, ApJ, 815, 102

Fong, W., Berger, E., Margutti, R., et al. 2012, ApJ, 756, 189

Fong, W., Berger, E., Metzger, B. D., et al. 2014, ApJ, 780, 118

Fox, D. B., Frail, D. A., Price, P. A., et al. 2005, Nature, 437, 845

Fryer, C. L. 2009, ApJ, 699, 409

Fryer, C. L., Belczynski, K., Ramirez-Ruiz, E., et al. 2015a, ApJ, 812,24

Fryer, C. L., Benz, W., \& Herant, M. 1996, ApJ, 460, 801

Fryer, C. L., Herwig, F., Hungerford, A., \& Timmes, F. X. 2006, ApJL, 646, L131

Fryer, C. L., Oliveira, F. G., Rueda, J. A., \& Ruffini, R. 2015b, Phys. Rev. Lett., 115, 231102

Fryer, C. L., Rueda, J. A., \& Ruffini, R. 2014, ApJ, 793, L36

Fryer, C. L., \& Woosley, S. E. 1998, ApJL, 502, L9

Fryer, C. L., Woosley, S. E., \& Hartmann, D. H. 1999a, ApJ, 526,152

Fryer, C. L., Woosley, S. E., Herant, M., \& Davies, M. B. 1999b, ApJ, 520, 650

Ghirlanda, G., Salafia, O. S., Pescalli, A., et al. 2016, A\&A, 594, A84

Giacconi, R., \& Ruffini, R., eds. 1978, Physics and astrophysics of neutron stars and black holes

Goldstein, A., Veres, P., Burns, E., et al. 2017, ApJL, 848, L14

Goodman, J. 1986, ApJL, 308, L47

Guetta, D., \& Della Valle, M. 2007, ApJL, 657, L73

Guetta, D., Pian, E., \& Waxman, E. 2011, A\&A, 525, A53

Haggard, D., Nynka, M., Ruan, J. J., et al. 2017, ApJL, 848, L25

Hills, J. G. 1983, ApJ, 267, 322

Izzo, L., Rueda, J. A., \& Ruffini, R. 2012a, A\&A, 548, L5

Izzo, L., Ruffini, R., Penacchioni, A. V., et al. 2012b, A\&A, 543, A10

Kawaguchi, K., Kyutoku, K., Nakano, H., et al. 2015, PhRvD, 92, 024014

Klein, A., Barausse, E., Sesana, A., et al. 2016, PhRvD, 93, 024003

Kluźniak, W., \& Ruderman, M. 1998, ApJL, 505, L113

Kobayashi, S., \& Mészáros, P. 2003, ApJ, 589, 861

Kovacevic, M., Izzo, L., Wang, Y., et al. 2014, A\&A, 569, A108

Landau, L. D., \& Lifshitz, E. M. 1975, Course of Theoretical Physics, Vol. Volume 2, The Classical Theory of Fields (Oxford: Pergamon Press)

Lazarus, P., Tauris, T. M., Knispel, B., et al. 2014, MNRAS, 437, 1485

Lee, W. H., Ramirez-Ruiz, E., \& Page, D. 2004, ApJL, 608, L5

Liang, E., Zhang, B., Virgili, F., \& Dai, Z. G. 2007, ApJ, 662, 1111

LIGO Scientific Collaboration, Aasi, J., Abbott, B. P., et al. 2015, Classical and Quantum Gravity, 32, 074001
MacFadyen, A. I., \& Woosley, S. E. 1999, ApJ, 524, 262

MacFadyen, A. I., Woosley, S. E., \& Heger, A. 2001, ApJ, 550, 410

Maggiore, M. 2007, Gravitational Waves. Vol. 1: Theory and Experiments (Oxford University Press)

Meszaros, P., \& Rees, M. J. 1997, ApJL, 482, L29

Mirabel, I. F., \& Rodríguez, L. F. 1998, Nature, 392, 673

Muccino, M., Ruffini, R., Bianco, C. L., Izzo, L., \& Penacchioni, A. V. 2013, ApJ, 763, 125

Narayan, R., Piran, T., \& Shemi, A. 1991, ApJL, 379, L17

Nicuesa Guelbenzu, A., Klose, S., Rossi, A., et al. 2011, A\&A, 531, L6

Paczynski, B. 1986, ApJL, 308, L43

Paschalidis, V., MacLeod, M., Baumgarte, T. W., \& Shapiro, S. L. 2009, PhRvD, 80, 024006

Patricelli, B., Razzano, M., Cella, G., et al. 2016, JCAP, 11, 056

Peters, P. C. 1964, Phys. Rev., 136, 1224

Peters, P. C., \& Mathews, J. 1963, Phys. Rev., 131, 435

Postnov, K. A., \& Yungelson, L. R. 2014, Living Reviews in Relativity, 17, 3

Rangel Lemos, L. J., Bianco, C. L., Mosquera Cuesta, H. J., Rueda, J. A., \& Ruffini, R. 2010, in 25th Texas Symposium on Relativistic Astrophysics, 204

Rees, M., Ruffini, R., \& Wheeler, J. A. 1974, Black holes, gravitational waves and cosmology (New York: Gordon and Breach Science Publishers Inc.)

Rhoades, C. E., \& Ruffini, R. 1974, Physical Review Letters, 32, 324

Rigault, M., Aldering, G., Kowalski, M., et al. 2015, ApJ, 802, 20

Rosswog, S., Ramirez-Ruiz, E., \& Davies, M. B. 2003, MNRAS, 345,1077

Rueda, J. A., \& Ruffini, R. 2012, ApJL, 758, L7

Ruffini, R., Bernardini, M. G., Bianco, C. L., et al. 2006, in The Tenth Marcel Grossmann Meeting, ed. M. Novello, S. Perez Bergliaffa, \& R. Ruffini (Singapore: World Scientific), 369

Ruffini, R., Bernardini, M. G., \& Bianco et al., C. L. 2008, in The Eleventh Marcel Grossmann Meeting, ed. H. Kleinert, R. T. Jantzen, \& R. Ruffini, 368-505

Ruffini, R., Bianco, C. L., Fraschetti, F., Xue, S.-S., \& Chardonnet, P. 2001, ApJL, 555, L117

Ruffini, R., \& Wilson, J. 1973, Phys. Rev. Lett., 31, 1362

Ruffini, R., Bernardini, M. G., Bianco, C. L., et al. 2007, in ESA Special Publication, Vol. 622, ESA Special Publication, 561

Ruffini, R., Izzo, L., Muccino, M., et al. 2014, A\&A, 569, A39

Ruffini, R., Wang, Y., Enderli, M., et al. 2015a, ApJ, 798, 10

Ruffini, R., Muccino, M., Kovacevic, M., et al. 2015b, ApJ, 808, 190

Ruffini, R., Muccino, M., Aimuratov, Y., et al. 2016a, ApJ, 831, 178

Ruffini, R., Rueda, J. A., Muccino, M., et al. 2016b, ApJ, 832, 136

Ruffini, R., Wang, Y., Aimuratov, Y., et al. 2018a, ApJ, 852, 53

Ruffini, R., Muccino, M., Aimuratov, Y., et al. 2018b, ArXiv e-prints, arXiv:1802.07552

Savchenko, V., Ferrigno, C., Kuulkers, E., et al. 2017, ApJL, 848, L15

Schaefer, B. E. 2007, ApJ, 660, 16

Shibata, M., \& Taniguchi, K. 2011, Living Rev. Relat., 14

Smartt, S. J. 2009, ARA\&A, 47, 63

-. 2015, PASA, 32, e016

Soderberg, A. M., Kulkarni, S. R., Nakar, E., et al. 2006a, Nature, 442, 1014

Soderberg, A. M., Berger, E., Kasliwal, M., et al. 2006b, ApJ, 650,261

Sun, H., Zhang, B., \& Li, Z. 2015, ApJ, 812, 33

Tauris, T. M., Langer, N., Moriya, T. J., et al. 2013, ApJL, 778, L23 
Tauris, T. M., Langer, N., \& Podsiadlowski, P. 2015a, MNRAS, 451, 2123

Tauris, T. M., van den Heuvel, E. P. J., \& Savonije, G. J. 2000, ApJL, 530, L93

Tauris, T. M., Kaspi, V. M., Breton, R. P., et al. 2015b, Advancing Astrophysics with the Square Kilometre Array (AASKA14), 39

Usov, V. V. 1992, Nature, 357, 472

Virgili, F. J., Liang, E.-W., \& Zhang, B. 2009, MNRAS, 392, 91

Virgili, F. J., Zhang, B., O'Brien, P., \& Troja, E. 2011, ApJ, 727, 109

Wanderman, D., \& Piran, T. 2010, MNRAS, 406, 1944
一. 2015, MNRAS, 448, 3026

Woosley, S. E. 1993, ApJ, 405, 273

Woosley, S. E., \& Bloom, J. S. 2006, ARA\&A, 44, 507

Yonetoku, D., Nakamura, T., Sawano, T., Takahashi, K., \& Toyanago, A. 2014, ApJ, 789, 65

Zel'dovich, Y. B., Ivanova, L. N., \& Nadezhin, D. K. 1972, Soviet Astronomy, 16, 209

Zhang, B., \& Mészáros, P. 2001, ApJL, 552, L35

Zhang, B., Zhang, B., Virgili, F. J., et al. 2009, ApJ, 703, 1696

Zhang, B.-B., Zhang, B., Sun, H., et al. 2017, ArXiv e-prints, arXiv:1710.05851 